\title{
Soil Reaction to Lateral Harmonic Pile Motion
}

\section{George Anoyatis}

Post-Doctoral researcher, Department of Civil and Environmental Engineering, Samueli School of Engineering, University of California Irvine, Irvine, CA

\section{George Mylonakis}

Chair in Geotechnics and Soil-Structure Interaction, Department of Civil Engineering, Faculty of Engineering, University of Bristol, Bristol UK

\section{Anne Lemnitzer}

(Corresponding Author)

Assistant Professor, Department of Civil and Environmental Engineering, Samueli School of Engineering, University of California Irvine, Irvine CA, Email: alemnitz@uci.edu

\begin{abstract}
The analytical representation of dynamic soil reaction to a laterally-loaded pile using 3D continuum modelling is revisited. The governing elastodynamic Navier equations are simplified by setting the dynamic vertical normal stresses in the soil equal to zero, which uncouples the equilibrium in vertical and horizontal directions and allows a closed-form solution to be obtained. This physically motivated approximation, correctly conforming to the existence of a free surface, was not exploited in earlier studies by Tajimi, Nogami and Novak and leads to a weaker dependence of soil response to Poisson's ratio which is in agreement with numerical solutions found in literature. The stress and displacement fields in the soil and the associated reaction to an arbitrary harmonic pile displacement are derived analytically using pertinent displacement potentials and eigenvalue expansions over the vertical coordinate. Both infinitely long piles and piles of finite length are considered. Results are presented in terms of dimensionless parameters and graphs that highlight salient aspects of the problem. A detailed discussion on wave propagation and cutoff frequencies based on the analytical findings is provided. A new dimensionless frequency parameter is introduced to demonstrate that the popular plane-strain model yields realistic values for soil reaction only at high frequencies and low Poisson's ratios.
\end{abstract}




\section{Introduction}

The degree of accuracy in predicting the lateral response of a pile subject to dynamic loading is strongly dependent on the reaction of the surrounding soil to the pile motion. Given its simplicity and versatility, the most well-known model capturing the soil reaction is described in the pioneering work of Baranov [1] and revisited by Novak [2]. The basic model assumes no variation in response along the vertical coordinate, hence treating the soil medium around the pile as a series of uncoupled incompressible horizontal slices in the analysis. Consequently, the Baranov-Novak model can be viewed as a plane strain case. Although this model yields soil reactions in closed form, it has been shown that reliable predictions are only obtained in the high frequency range.

To address the limitation identified above, the current study seeks to provide a solution that builds upon the work of Tajimi [3], Nogami and Novak [4], and Saitoh and Watanabe [5] which handle the problem in three dimensions. Our pile is considered a vertical cylinder and the soil is modelled as a continuum, taking into account all three components of soil displacement under the assumption of zero dynamic vertical normal stresses. A similar assumption was adopted in earlier studies [6] [7], however, proposed solutions were limited to the kinematic response of laterally loaded piles. This physically motivated simplification is particularly attractive, as it respects the boundary condition associated with the presence of a stress - free soil surface and reduces the number of governing equations to two - instead of three as found in the classical elastodynamic theory ( [8], [9]). Contrary to early studies where the vertical soil displacement was set equal to zero ( [10], [3], [4]), in this work the assumption will be less restrictive: vertical soil displacement is small, yet not zero. As shown in the following, this approach overcomes the singularities of earlier models in the important case of incompressible soil.

The equations of motion in the soil medium are then solved analytically through pertinent eigen-expansions. Closed-form solutions as a function of pile displacement amplitude are obtained for the displacement field in the soil and the soil reaction to lateral pile motion. The soil reaction is expressed in terms of a dimensionless soil reaction factor $\left(R^{*}\right)$ which depends on pile slenderness, soil material damping, Poisson's ratio and excitation frequency. The effect of these parameters on the reaction factor are explored analytically and presented in dimensionless graphs. In the dynamic regime, the real part of the reaction factor describes the stiffness of the soil layer and the imaginary part describes the corresponding damping. The reaction of the soil layer can be directly employed in the solution of the soil-pile interaction problems based on the boundary conditions at the pile head and tip, and can be easily calculated for any given pile displacement profile [11]. Soil-pile interaction analysis has also been undertaken and will be presented in a companion study. Empirical expressions for dynamic soil reaction to lateral pile motion have been proposed, among others, by Roesset [12], Dobry et al. [13], Gazetas and Dobry [14] [15], and Mylonakis 
[16]. Summaries of available information are provided in Tajimi [17], Pender [18], Gazetas and Mylonakis [19], Syngros [20], Guo [21] and more recently by Saitoh and Watanabe [5] and Shadlou and Bhattacharya [22]. Additional information on the Tajimi model is provided by Akiyoshi [23], Veletsos and Younan [6], Chau and Yang [24], and Latini et al. [25].

The scope of this paper is multi-fold: (i) to review the plane strain model of Baranov - Novak and discuss under which conditions its predictions are realistic; (ii) to derive an improved three-dimensional elastodynamic solution with an emphasis on the performance at high values of soil Poisson's ratio; (iii) to explain the wave propagation mechanisms that develop in soil under the influence of harmonic lateral pile oscillations; (iv) to extend the solution to the important case of an infinitely long pile; (v) to provide novel normalization schemes for soil reaction that lead to results approaching a single master curve, as well as simplified formulae that can be used in applications. Upon successful derivation, the improved model will be used in a follow-up paper to analyze the lateral harmonic oscillations of a single pile.

\section{Problem Definition}

The soil - pile system considered in this study is described in cylindrical coordinates as shown in Fig. 1: a vertical cylindrical pile is embedded in a homogeneous soil layer overlying a rigid bedrock and is subjected to a harmonic lateral movement $w(z, t)=w(z, \omega) e^{i \omega t}$, where $t$ is the time variable, $\omega$ is the cyclic excitation frequency and $i$ is the imaginary number $(i=\sqrt{-1})$. The soil layer of thickness $H$ is treated as a continuum and is described by its Young's modulus $E_{s}$, mass density $\rho_{s}$ and Poisson's ratio $v_{s}$. In dynamic analyses the soil is treated as a dissipative material with hysteretic damping $\beta_{s}$ expressed through a complex - valued Young's modulus $E_{s}^{*}=E_{s}\left(1+2 i \beta_{s}\right)$. Note that for the problem at hand a perfectly bounded interface between pile and soil is assumed, although this assumption can be relaxed [26].

\section{Model Development}

The equilibrium of forces acting on an arbitrary soil element along the radial and tangential direction is expressed in terms of Cauchy stresses as:

$$
\frac{\partial \sigma_{r}}{\partial r}+\frac{\partial \tau_{r z}}{\partial z}+\frac{1}{r} \frac{\partial \tau_{r \theta}}{\partial \theta}+\frac{1}{r}\left(\sigma_{r}-\sigma_{\theta}\right)-\rho_{s} \frac{\partial^{2} u_{r}}{\partial t^{2}}=0
$$


$\frac{\partial \tau_{r \theta}}{\partial r}+\frac{\partial \tau_{\theta z}}{\partial z}+\frac{1}{r} \frac{\partial \sigma_{\theta}}{\partial \theta}+\frac{2}{r} \tau_{r \theta}-\rho_{s} \frac{\partial^{2} u_{\theta}}{\partial t^{2}}=0$

where $u_{r}=u_{r}(r, \theta, z, t)$ and $u_{\theta}=u_{\vartheta}(r, \theta, z, t)$ are the horizontal and tangential soil displacements, $\sigma_{r}=\sigma_{r}(r, \theta, z, t)$ and $\sigma_{\theta}=\sigma_{\theta}(r, \theta, z, t)$ are the normal stresses acting along $r$ and $\theta$, respectively; $\tau_{r z}=\tau_{r z}(r, \theta, z, t)$ is the shear stress along $z$ and perpendicular to $r ; \tau_{r \theta}=\tau_{r \theta}(r, \theta, z, t)$ is the shear stress along $\theta$ and perpendicular to $r$; and $\tau_{\theta z}=\tau_{\theta z}(r, \theta, z, t)$ is the shear stress along $z$ and perpendicular to $\theta$. Note that the last term in each equation associated with the soil mass density $\rho_{s}$ is accounting for the inertia of the soil in radial and tangential direction under dynamic excitation.

Taking into account stress - strain relations in cylindrical coordinates (see Appendix A) and considering harmonic soil response of the form $u_{r}=u_{r}(r, \theta, z, \omega) e^{i \omega t}$ and $u_{\theta}=u_{\theta}(r, \theta, z, \omega) e^{i \omega t}$, Eqs (1) can be rewritten in terms of displacements in the following form:

$$
\begin{aligned}
& \eta_{s}^{2} \frac{\partial}{\partial r}\left[\frac{1}{r}\left(\frac{\partial\left(r u_{r}\right)}{\partial r}+\frac{\partial u_{\theta}}{\partial \theta}\right)\right]-\frac{1}{r^{2}} \frac{\partial}{\partial \theta}\left[\frac{\partial\left(r u_{\theta}\right)}{\partial r}-\frac{\partial u_{r}}{\partial \theta}\right]+\frac{\partial^{2} u_{r}}{\partial z^{2}}+\left(\frac{\omega}{V_{s}^{*}}\right)^{2} u_{r}=0 \\
& \frac{\partial}{\partial r}\left[\frac{1}{r}\left(\frac{\partial\left(r u_{\theta}\right)}{\partial r}-\frac{\partial u_{r}}{\partial \theta}\right)\right]+\eta_{s}^{2} \frac{1}{r^{2}} \frac{\partial}{\partial \theta}\left[\frac{\partial\left(r u_{r}\right)}{\partial r}+\frac{\partial u_{\theta}}{\partial \theta}\right]+\frac{\partial^{2} u_{\theta}}{\partial z^{2}}+\left(\frac{\omega}{V_{s}^{*}}\right)^{2} u_{\theta}=0
\end{aligned}
$$

where $V_{s}^{*}=V_{s} \sqrt{1+2 i \beta_{s}}$ is the complex - valued shear wave propagation velocity in the soil.

In the above equations $\eta_{s}$ is a dimensionless compressibility coefficient which is solely a function of soil Poisson's ratio and is associated with the effect of vertical soil displacement on stresses. It is noted that unlike the vertical response mode where a number of stress-displacement terms are set equal to zero in a corresponding formulation [27], in the solution at hand setting $\sigma_{z}$ equal to zero is the only approximation involved. It is also worth mentioning that due to this approximation shear stress $\tau_{r z}$ ceases to be zero at the soil surface, yet this violation typically has a minor effect on the solution [6], [7], [11]. Additional discussion is provided in the ensuing.

Following Graff [8], a degenerate Helmholtz decomposition scheme is applied to uncouple the above set of partial differential equations. To this end, horizontal and tangential displacements are expressed in terms of two potential functions $\boldsymbol{\Phi}$ and $\boldsymbol{\Psi}$ as shown below:

$$
\begin{aligned}
& u_{r}=\frac{\partial \Phi}{\partial r}+\frac{1}{r} \frac{\partial \Psi}{\partial \theta} \\
& u_{\theta}=\frac{1}{r} \frac{\partial \Phi}{\partial \theta}-\frac{\partial \Psi}{\partial r}
\end{aligned}
$$


Substituting Eqs (3) in (2) leads to the following new set of uncoupled differential equations

$$
\begin{gathered}
\eta_{s}^{2} \nabla^{2} \boldsymbol{\Phi}+\frac{\partial^{2} \boldsymbol{\Phi}}{\partial z^{2}}+\left(\frac{\omega}{V_{s}^{*}}\right)^{2} \boldsymbol{\Phi}=0 \\
\nabla^{2} \boldsymbol{\Psi}+\frac{\partial^{2} \boldsymbol{\Psi}}{\partial z^{2}}+\left(\frac{\omega}{V_{s}^{*}}\right)^{2} \boldsymbol{\Psi}=0
\end{gathered}
$$

where $\nabla^{2}=\frac{\partial^{2}}{\partial r^{2}}+\frac{1}{r} \frac{\partial}{\partial r}+\frac{1}{r^{2}} \frac{\partial^{2}}{\partial \theta^{2}}$ is the Laplacian operator. The solution to the above equations can be obtained using the method of separation of variables which allows the transformation of the partial differential equations into a set of ordinary differential equations which are easier to handle. In the realm of this approach, potential functions $\boldsymbol{\Phi}$ and $\boldsymbol{\Psi}$ can be expressed as products of three modular functions i.e., $\boldsymbol{\Phi}(r, \theta, z)=R_{1}(r) \Theta_{1}(\theta) Z_{1}(z)$ and $\boldsymbol{\Psi}(r, \theta, z)=R_{2}(r) \Theta_{2}(\theta) Z_{2}(z)$, which yield the following equations:

$$
\begin{aligned}
& \eta_{s}^{2} \frac{1}{R_{1}}\left[\frac{d^{2} R_{1}}{d r^{2}}+\frac{1}{r} \frac{d R_{1}}{d r}\right]+\eta_{s}^{2} \frac{1}{r^{2}} \frac{1}{\Theta_{1}} \frac{d \Theta_{1}^{2}}{d \theta^{2}}+\frac{1}{Z_{1}} \frac{d Z_{1}^{2}}{d z^{2}}+\left(\frac{\omega}{V_{s}^{*}}\right)^{2}=0 \\
& \frac{1}{R_{2}}\left[\frac{d^{2} R_{2}}{d r^{2}}+\frac{1}{r} \frac{d R_{2}}{d r}\right]+\frac{1}{r^{2}} \frac{1}{\Theta_{2}} \frac{d \Theta_{2}^{2}}{d \theta^{2}}+\frac{1}{Z_{2}} \frac{d Z_{2}^{2}}{d z^{2}}+\left(\frac{\omega}{V_{s}^{*}}\right)^{2}=0
\end{aligned}
$$

The above mathematical representation leads to the decomposition of the partial differential equations into three ordinary differential equations in $r, \theta$ and $z$ :

$$
\begin{aligned}
& \frac{d^{2} R}{d r^{2}}+\frac{1}{r} \frac{d R}{d r}-\left(q^{2}+\frac{n^{2}}{r^{2}}\right) R=0 \\
& \frac{d^{2} \Theta}{d \theta^{2}}+n^{2} \Theta=0 \\
& \frac{d^{2} Z}{d z^{2}}+a^{2} Z=0
\end{aligned}
$$

from which it arises that

$$
q=\frac{1}{\eta_{s}} \sqrt{a^{2}-\left(\frac{\omega}{V_{s}^{*}}\right)^{2}}
$$


$a$ and $n$ being positive real numbers; $q$ has dimensions of $1 /$ Length and can be viewed as an attenuation parameter (wavenumber) for radially propagating waves. Upon obtaining the general solutions to the above equations ( [28]), the potential functions are written as follows:

$$
\begin{aligned}
& \Phi=\left[A_{1} I_{n}(q r)+B_{1} K_{n}(q r)\right]\left[A_{2} \sin n \theta+B_{2} \cos n \theta\right]\left[A_{3} \sin a z+B_{3} \cos a z\right] \\
& \Psi=\left[A_{4} I_{n}\left(q \eta_{s} r\right)+B_{4} K_{n}\left(q \eta_{s} r\right)\right]\left[A_{5} \sin n \theta+B_{5} \cos n \theta\right]\left[A_{6} \sin a z+B_{6} \cos a z\right]
\end{aligned}
$$

where $I_{n}()$ and $K_{n}()$ are the modified Bessel functions of the $n$-th order and the first and second kind, respectively. $A_{i}, B_{i}(i=1,2,3, \ldots, 6)$ are constants to be determined from the boundary conditions of the problem.

To ensure bounded response at large radial distances from the pile $(r \rightarrow \infty)$, constants $A_{1}$ and $A_{4}$ associated with the modified Bessel functions $I_{n}($ ) must vanish. Considering the direction of pile loading to be along $\theta=0$, constants $A_{2}$ and $B_{5}$ must vanish as well to satisfy the conditions of zero radial and tangential displacement components, $u_{r}$ and $u_{\theta}$, at $\theta=\pi / 2$ and $\theta=0$, respectively. This eliminates the trigonometric functions $\sin ()$ and $\cos ()$ in Eqs (8a) and (8b), respectively. This is in accordance with a positive displacement $u_{r}\left(u_{r}>0\right)$ in the range $-\pi / 2 \leq \theta \leq \pi / 2$ and a negative displacement $\left(u_{r}<0\right)$ in the range $\pi / 2 \leq \theta \leq 3 \pi / 2$. Likewise, the tangential displacement is positive $\left(u_{\theta}>0\right)$ in the range $0 \leq$ $\theta \leq \pi$ and negative $\left(u_{\theta}<0\right)$ in the range $\pi \leq \theta \leq 2 \pi$. The above are valid for $n=1$.

The additional conditions of zero soil displacements at the base of the soil layer and stress-free soil surface $\left(\tau_{r \theta}=0\right)$ enforce $A_{3}=A_{6}=0$ and $\cos a H=0$ which, in turn, yields

$a_{m}=\frac{\pi}{2 H}(2 m-1), \quad m=1,2,3, \ldots$

where $a_{m}$ are the eigenvalues of the system with $a_{1}<a_{2}<a_{3} \ldots<a_{N}$ ( $N$ being the total number of modes employed in the analysis).

In light of the above, Eqs (8) simplify to

$$
\begin{aligned}
& \Phi_{m}=B_{2} \cos \theta K_{1}\left(q_{m} r\right) \sin a_{m} z \\
& \Psi_{m}=A_{5} \sin \theta K_{1}\left(q_{m} \eta_{s} r\right) \sin a_{m} z
\end{aligned}
$$

The differential equation with respect to $z$ (Eq. 6c) is recognized as a Sturm - Liouville (S-L) equation with constant coefficients. Since parameter $a$ is not specified, finding the values for which nontrivial solutions exist is part of the S-L theory. Such values are called the eigenvalues of the boundary-value problem and 
the corresponding solutions for function $Z(z)$ are the eigenfunctions (i.e., the "soil modes"). It is important to note that these modes are not necessarily associated with dynamic soil response (i.e., they are merely an orthogonal set of functions) and exist even in the static case. Corresponding to each eigenvalue $a_{m}$ a unique eigenfunction $\Phi_{m}(z)$ exists which is called the $m$-th fundamental solution. For the current problem the trigonometric functions $\sin a_{m} z$ are the normal modes (eigenfunctions)

$$
\Phi_{m}(z)=\sin a_{m} z
$$

which satisfy the ordinary differential equation (Eq. 6c) with respect to the spatial variable $z$ and the boundary conditions of the problem. The first five modes of vibration are depicted in Fig. 2, where the points of zero displacement are the nodes of vibration and the points of maximum vibration are the corresponding antinodes.

The normal modes have the important property of orthogonality which is described mathematically as follows

$$
\int_{0}^{H} \Phi_{k}(z) \Phi_{m}(z) d z=0, \quad m \neq k \quad(m, k=1,2,3, \ldots)
$$

It is anticipated that the solution should be obtained by the superposition of all particular solutions (modes). Thus soil displacement components $u_{r}$ and $u_{\theta}$ should be expressed as an infinite sum of Fourier terms including the soil modes $\Phi_{m}$ and a term $U_{r, \theta} m$ associated with the spatial variable $r$. Accordingly,

$$
u_{r, \theta m} \propto \sum_{m=1}^{\infty} U_{r, \theta m}(r, \omega) \Phi_{m}(z)
$$

The solution will be obtained as a superposition of the particular solutions ("modes", Eqs 15). Since soil modes form an orthogonal set, pile displacement $w$ can be expressed through a normal-mode expansion similar to soil response (Eq. 13)

$$
w(z, \omega)=\sum_{m=1}^{\infty} W_{m}(\omega) \Phi_{m}(z)
$$

where the frequency - varying coefficients $W_{m}$ are measured in units of length. Substituting Eq. (10) into (3), imposing compatibility of displacements in horizontal and tangential direction i.e., $u_{r}(d / 2,0, z, \omega)=w(z, \omega)$ and $u_{\theta}(d / 2, \pi / 2, z, \omega)=-w(z, \omega)$ and taking into account the orthogonality of soil modes, displacement components $u_{r}, u_{\theta}$ are obtained as 


$$
\begin{aligned}
& u_{r}(r, \theta, \mathrm{z}, \omega)=\cos \theta \sum_{m=1}^{\infty} U_{r m}(r, \omega) W_{m}(\omega) \Phi_{m}(z) \\
& u_{\theta}(r, \theta, \mathrm{z}, \omega)=\sin \theta \sum_{m=1}^{\infty} U_{\theta m}(r, \omega) W_{m}(\omega) \Phi_{m}(z)
\end{aligned}
$$

which satisfy the symmetry conditions $u_{r}(r,-\pi / 2, z)=u_{r}(r, \pi / 2, z)$ and $u_{r}(r, 0, z)=u_{r}(r, \pi, z)$, where

$$
\begin{aligned}
& U_{r m}(r, \omega)=B_{m}\left[s_{m} K_{0}\left(q_{m} r\right)+\left(\frac{d}{2 r}\right) K_{1}\left(q_{m} r\right)\right]-A_{m}\left(\frac{d}{2 r}\right) K_{1}\left(q_{m} \eta_{s} r\right) \\
& U_{\theta m}(r, \omega)=B_{m}\left(\frac{d}{2 r}\right) K_{1}\left(q_{m} r\right)-A_{m}\left[s_{m} \eta_{s} K_{0}\left(q_{m} \eta_{s} r\right)+\left(\frac{d}{2 r}\right) K_{1}\left(q_{m} \eta_{s} r\right)\right]
\end{aligned}
$$

$A_{m}$ and $B_{m}$ being the dimensionless constants

$$
\begin{aligned}
& A_{m}=\frac{2 K_{1}\left(s_{m}\right)+s_{m} K_{0}\left(s_{m}\right)}{s_{m} K_{0}\left(s_{m}\right) K_{1}\left(s_{m} \eta_{s}\right)+s_{m} \eta_{s} K_{0}\left(s_{m} \eta_{s}\right)\left[s_{m} K_{0}\left(s_{m}\right)+K_{1}\left(s_{m}\right)\right]} \\
& B_{m}=\frac{2 K_{1}\left(s_{m} \eta_{s}\right)+s_{m} \eta_{s} K_{0}\left(s_{m} \eta_{s}\right)}{s_{m} K_{0}\left(s_{m}\right) K_{1}\left(s_{m} \eta_{s}\right)+s_{m} \eta_{s} K_{0}\left(s_{m} \eta_{s}\right)\left[s_{m} K_{0}\left(s_{m}\right)+K_{1}\left(s_{m}\right)\right]}
\end{aligned}
$$

where $s_{m}=q_{m} d / 2$ (Eq. 7) is a dimensionless parameter.

\section{Compressibility coefficient}

Mention has already been made to the study of Nogami and Novak [4] where the vertical soil displacement $u_{z}$ was set equal to zero and, thus, the corresponding normal strain was $\varepsilon_{z}=0$. This assumption leads to the following expression for the compressibility factor

$\eta_{s}=\sqrt{\frac{2\left(1-v_{s}\right)}{1-2 v_{s}}}$

which expresses the square root of the ratio of the constrained modulus $M$ (P-wave modulus) to the shear modulus of the soil material (i.e., $\eta_{s}=\sqrt{M / G_{S}}$ ). A problem arising from the use of this equation is the sensitivity to Poisson's ratio, as $\eta_{s}$ becomes infinitely large when $v_{s}$ approaches 0.5 . This behavior is spurious (e.g. leads to zero values of wavenumber q in Eq. 7) and has not been observed in rigorous numerical solutions of such problems, e.g., [29], [30], [6]. It is important to note that despite that $u_{z}$ is 
assumed to be zero, the specific solution is still three dimensional (i.e. not plane strain) as the variation of the response with respect to the vertical coordinate $(z)$ is finite - due to the shear coupling among the various slices.

The alternative assumption of $\sigma_{z}=0$, [11], which complies with a stress-free soil surface leads to finite vertical displacement which yields:

$\eta_{s}=\sqrt{\frac{2-v_{s}}{1-v_{s}}}$

Equations (18) and (19) are compared graphically in Fig. 3. Except where specifically indicated otherwise, the solutions presented hereafter are based on Eq. (19).

\section{Soil Reaction}

At the soil-pile interface, the amplitude of horizontal soil reaction $p(z, \omega)$ resulting from the pile motion is expressed as [4], [6], [26]

$$
p(z, \omega)=-\int_{0}^{2 \pi}\left[\sigma_{r, 0} \cos \theta-\tau_{r \theta, 0} \sin \theta\right](d / 2) d \theta
$$

where $\sigma_{r, 0}=\sigma_{r}(d / 2, \theta, z, \omega)$ and $\tau_{r \theta, 0}=\tau_{r \theta}(d / 2, \theta, z, \omega)$ are the maximum radial normal stress and shear stress, respectively, acting at the periphery of the pile

$$
\sigma_{r, 0}=\cos \theta \frac{2 G_{s}^{*}}{(d / 2)} \sum_{m=1}^{\infty} S_{m}(\omega) W_{m}(\omega) \Phi_{m}(z)
$$

and

$$
\tau_{r \theta, 0}=\sin \theta \frac{2 G_{s}^{*}}{(d / 2)} \sum_{m=1}^{\infty} T_{m}(\omega) W_{m}(\omega) \Phi_{m}(z)
$$

where the dimensionless coefficients $S_{m}$ and $T_{m}$ are given by the following expressions:

$$
\begin{aligned}
& S_{m}=-B_{m}\left[s_{m} K_{0}\left(s_{m}\right)+\frac{s_{m}^{2} \eta_{\sigma}^{2}}{2} K_{1}\left(s_{m}\right)+2 K_{1}\left(s_{m}\right)\right]+A_{m}\left[s_{m} \eta_{s} K_{0}\left(s_{m} \eta_{s}\right)+2 K_{1}\left(s_{m} \eta_{s}\right)\right] \\
& T_{m}=-B_{m}\left[s_{m} K_{0}\left(s_{m}\right)+2 K_{1}\left(s_{m}\right)\right]+A_{m}\left[s_{m} \eta_{s} K_{0}\left(s_{m} \eta_{s}\right)+\frac{s_{m}^{2} \eta_{s}^{2}}{2} K_{1}\left(s_{m} \eta_{s}\right)+2 K_{1}\left(s_{m} \eta_{s}\right)\right]
\end{aligned}
$$


Substituting the above expressions into Eq. (20) and noting that $\int_{0}^{2 \pi} \cos ^{2} \theta d \theta=\int_{0}^{2 \pi} \sin ^{2} \theta d \theta=\pi$, the horizontal soil reaction can be written in the alternative form

$$
p(z, \omega)=\pi G_{s}^{*} \sum_{m=1}^{\infty} R_{m}^{*}(\omega) W_{m}(\omega) \Phi_{m}(z)
$$

where $R_{m}^{*}$ is a complex valued soil reaction factor associated with the $m$-th soil mode:

$$
R_{m}^{*}=s_{m}^{2} \frac{\eta_{\sigma}^{2}\left[2 K_{1}\left(s_{m} \eta_{s}\right)+s_{m} \eta_{s} K_{0}\left(s_{m} \eta_{s}\right)\right] K_{1}\left(s_{m}\right)+\eta_{s}^{2}\left[2 K_{1}\left(s_{m}\right)+s_{m} K_{0}\left(s_{m}\right)\right] K_{1}\left(s_{m} \eta_{s}\right)}{s_{m} K_{0}\left(s_{m}\right) K_{1}\left(s_{m} \eta_{s}\right)+s_{m} \eta_{s} K_{0}\left(s_{m} \eta_{s}\right)\left[s_{m} K_{0}\left(s_{m}\right)+K_{1}\left(s_{m}\right)\right]}
$$

$\eta_{\sigma}$ in Eq. (25) is denoted the secondary compressibility coefficient and is expressed as:

$\eta_{\sigma}=\sqrt{\frac{2}{1-v_{s}}}$

Note that Eq. (26) corresponds to the compressibility factor $\eta_{s}$ adopted by Anoyatis and Mylonakis [27] for the axially-load problem. It is also important to note that in the alternative solution of Nogami and Novak [4] the reaction factor $R_{m}^{*}$ is obtained from the above expression using $\eta_{\sigma}=\eta_{s}$, where $\eta_{s}$ is given from Eq. (18). In both solutions the soil reaction factor depends mainly on soil parameters (i.e., $v_{s}, \beta_{s}, V_{s}, G_{s}$ ), the excitation frequency $\omega$ and on only one pile parameter, which is the diameter $d$.

In the dynamic regime, the complex-valued soil reaction factor can be cast in the following equivalent forms

$$
R_{m}^{*}=\operatorname{Real}\left(R_{m}^{*}\right)+i \operatorname{Imaginary}\left(R_{m}^{*}\right)=R_{m}(\omega)\left(1+2 i \beta_{m}\right)
$$

where $R_{m}(\omega)=\operatorname{Real}\left(R_{m}^{*}\right)$ is the dynamic storage stiffness and $2 \beta_{m} R_{m}(\omega)=\operatorname{Imaginary}\left(R_{m}^{*}\right)$ is the corresponding loss stiffness. The dimensionless parameter $\beta_{m}=\operatorname{Imaginary}\left(R_{m}^{*}\right) / 2 \operatorname{Real}\left(R_{m}^{*}\right) \operatorname{defines}$ an equivalent damping ratio, which is analogous to percentage of critical damping in a simple oscillator (Clough and Penzien [31]). $R_{m}(\omega)$ can be interpreted as a frequency dependent spring and $2 \beta_{m} R_{m}(\omega) / \omega$ as a dashpot attached in parallel to the spring. Note that the damping ratio $\beta_{m}$ is different from the soil material damping $\beta_{s}$, and can be alternatively expressed as $\beta_{m}=\beta_{s}+\left(\beta_{r}\right)_{m}$, where $\left(\beta_{r}\right)_{m}$, is the radiation damping ratio associated with the $m$-th propagating mode. $\beta_{m} \approx \beta_{s}$ is a special case which is valid only for frequencies in the range $0<\omega<\omega_{m}$. 
Recalling Eq. (25) soil reaction is expressed as $p(z, \omega)=\pi \sum R_{m} G_{s} w(z)$. This shows that $R_{m}$ can be viewed as a dimensionless factor which modifies the soil stiffness $G_{S}$ due to the presence of the pile. In addition, it is clear that $R_{m}$ is mode-dependent which indicates that each mode has a different influence on the soil stiffness. Expressing the soil reaction in the simple form $p=k w$ [ $k$ can be viewed as a Winkler modulus (in units of force per length square)] allows us to write $k=\left(\pi R_{m}\right) G_{s}$, which shows that an average depth-independent Winkle modulus multiplied by the profile of displacement at the soil-pile interface yields the soil reaction profile.

\section{Wave Propagation}

When a pile oscillates energy is transmitted to the soil. Part of this energy is stored in the soil in the form of dynamic deformations and the remaining part is lost, e.g., being transformed into heat (material damping) or radiating to infinity in the form of stress waves (radiation damping). An initial discussion presented below treats the vertical and horizontal direction separately - investigating the contribution of each mode to the wave propagation, followed by a presentation of the combined wave effect.

\subsection{Vertical direction}

In vertical direction wave propagation is associated with the term $\sin a_{m} z e^{i \omega t}$. This term describes an oscillating motion of the $m$-th mode, which is of a stationary nature in space (i.e., along the vertical axis $z$ ) and varies with time $t$. Using trivial mathematical procedures, the above term may be rewritten in the alternative form $i\left(e^{-i\left(a_{m} z-\omega t\right)}-e^{i\left(a_{m} z+\omega t\right)}\right) / 2$. This equivalent representation shows that each exponent represents a disturbance propagating in the vertical direction. The first exponent describes a wave travelling from the soil surface to the base, while the second term is associated with a wave that follows the reverse path. Using the principle of superposition these two opposite directional waves which have the same frequency $\omega$, wavenumber $a_{m}$ and amplitude form a standing wave. This wave does not travel vertically, but stands still and oscillates horizontally. Hereby each mode $m$ forms a vertically varying disturbance (dynamic deformation) which varies with time. This not a travelling wave, thus no loss of energy in terms of radiation damping occurs.

It can be easily deduced that at depths $Z_{m, k}=2 H(k-1) /(2 m-1)$ the amplitude of the motion is always zero for the mode examined and these points are called the nodes. Note that the index $m$ refers to the $m$-th mode and the index $k$ to the number of nodes which appear in length $H$ of the same mode. Evidently, only one node exists at $z=0$ for the 1 -st mode $(m=1)$ whereas for the 2-nd mode $(m=2)$, two nodes appear 
at $z=0$ and $z=2 H / 3$, and so on (Fig. 2). Due to the boundary condition at the base, the location $z=0$ represents a node in all modes.

Analogously, depths described by $Z_{m, k}=H(2 k-1) /(2 m-1)$ are called antinodes and the wave amplitude is maximum. The distance between two successive nodes or antinodes is equal to the wavelength $2 \pi / a_{m}$ or $4 H /(2 m-1)$ for each mode. Note that when rewriting the superscript of the exponential functions as $i a_{m}\left(z+V_{m} t\right)$ or $-i a_{m}\left(z-V_{m} t\right)$, the term $V_{m}=\omega / a_{m}$ with dimensions of velocity arises, which stands for the phase velocity of the wave associated with a given mode $m$. This indicates that for a given frequency $\omega$ each harmonic wave propagates with a mode-specific velocity which is different for each mode. This reveals the development of a distortion mechanism called modal dispersion as the propagation velocity is not the same for all modes.

In conclusion, at each depth in the soil layer influx and efflux of energy occurs due to upward and downward travelling waves. For each mode these opposite directional waves form a standing wave, which is characterized by nodes and anti-nodes. At the nodes influx and efflux of kinetic energy is balanced, hence no motion occurs. Nevertheless, strain still develops and changes with time. The response of the soil is computed by superposition of $N$ modes (or a superposition of $N$ standing waves). Consequently motion develops at all depths.

\section{$\underline{5.2 \text { Horizontal direction }}$}

In the horizontal direction the wave propagation is associated with the term $U_{r, \theta m}$ (Eqs 13, 15) and is mathematically represented through the Bessel functions $K_{0}()$ and $K_{1}()$ and their argument $q_{m}$ (Eq. 7). The variation of $q_{m}$ with frequency is schematically illustrated in Figs 4 and 5 for an undamped $\left(\beta_{s}=0\right)$ and a damped $\left(\beta_{s} \neq 0\right)$ medium.

In absence of soil material damping (i.e., for an undamped medium as shown in Fig. 4a), Eq. (7) may be rewritten as

$q_{m}=\frac{1}{\eta_{s} V_{s}} \sqrt{\omega_{m}^{2}-\omega^{2}}$

where $\omega_{m}=(2 m-1) \omega_{1}$ and $\omega_{1}=\pi V_{s} / 2 H$ are the $m$-th and 1 -st resonant frequencies of the system, respectively.

For $\omega<\omega_{m}, q_{m}$ is real valued and decreases with increasing frequency. At $\omega=\omega_{m}, q_{m}$ drops to zero and a further increase in frequency $\left(\omega>\omega_{m}\right)$ yields purely imaginary wavenumbers $q_{m}=i \bar{q}_{m}$, which increase with increasing frequency $\left(\bar{q}_{m}\right.$ being a real number). $\omega_{m}$ represent the transition from propagation to non- 
propagation and is called the cutoff frequency of the $m$-th mode or $m$-th resonant frequent of the system. Note that $\omega_{1}$ coincides with the natural frequency of the deposit in shearing oscillations. No wave propagation (i.e., radiation of energy) is observed for lower frequencies. As shown later, $\omega_{1}$ is associated with a significant drop in stiffness and an increase in damping due to the emergence of travelling waves. For $\omega<\omega_{m}, U_{r, \theta m}($ Eqs 13,15) is responsible for a monotonic decrease in soil response with increasing horizontal distance from the pile axis and is not associated with wave propagation in the medium. For $\omega>$ $\omega_{m}$, stress waves associated with the $m$-th mode emerge from the soil-pile interface and propagate horizontally in the soil medium. At $\omega=\omega_{m}, q_{m}=0$ and yields an infinite wavelength $\lambda\left(=2 \pi / q_{m}\right)$ which for a finite frequency $\omega_{m}$ yields an infinite phase velocity $V\left(=\omega / q_{m}\right)$. This indicates that at any resonant frequency $\omega_{m}$ there is no spatial variation in motion in $r$ direction for the $m$-th mode. Since $U_{r, \theta m}\left(\omega=\omega_{m}\right)=1$ (Eqs 15), the system undergoes harmonic motions and the contribution of the $m$-th mode to the soil vibration can be expressed using the following simplified equation:

$u_{r, \theta m}\left(\omega=\omega_{m}\right)=(\cos \theta, \sin \theta) W_{m}(\omega) \Phi_{m}(z) e^{i \omega t}$

In presence of material damping $\beta_{s}$, in the soil (i.e., damped medium as shown in Fig. 4b), Eq. (7) may be approximated as follows:

$$
q_{m} \approx \frac{1}{\eta_{s} V_{s}} \sqrt{\underbrace{\left(\omega_{m}^{2}-\omega^{2}\right)}_{\begin{array}{c}
\text { "real } \\
\text { part" }
\end{array}}+i 2 \beta_{s} \omega}
$$

Contrary to the undamped medium, the above equation yields non-real-valued wavenumbers $q_{m}$, even in the frequency range of $\omega<\omega_{m}$. Complex valued wavenumbers for damped soils cause a monotonic reduction in soil displacement with radial distance, which is found to be stronger than in an undamped medium. At $\omega \approx \omega_{m}, \operatorname{Real}\left(q_{m}\right)$ drops to a minimum (non-zero) value. For $\omega>\omega_{m}$ the trend is reversed and $\operatorname{Real}\left(q_{m}\right)$ increases with frequency. On the other hand, $\operatorname{Imaginary}\left(q_{m}\right)$ always increases with frequency. As in the case of an undamped medium, for $\omega>\omega_{m}$ waves associated with the $m$-th mode start to emerge from the soil-pile interface and propagate horizontally in the soil medium, while $q_{m}$ is complex valued instead of purely imaginary.

Figure 5 presents the frequency spectrum for the first five modes for an undamped medium and a medium with soil damping $\beta_{s}=0.05$. Assuming that the complex valued $q_{m}$ can be written as $q_{m}+i \bar{q}_{m}$, it becomes evident that for a given frequency $q_{1}<q_{2}<\cdots<q_{5}$ and $\bar{q}_{1}>\bar{q}_{2}>\cdots>\bar{q}_{5}$, for both, an undamped $\left(\beta_{s}=0\right)$ and a damped $\left(\beta_{s}=0.05\right)$ medium. 
Undamped medium (Fig. 5): At $\omega=\omega_{1}, q_{1}=0$ while all $q_{1+m}$ pertaining to higher modes attain positive real values. Further increase in frequency $\left(\omega_{1}<\omega<\omega_{2}\right)$ yields a purely imaginary $q_{1}=i \bar{q}_{1}$, whereas the rest of wavenumbers $q_{1+m}$ remain real valued. Now the Bessel functions $K()$ of complex argument represent travelling waves in the radial direction - with an in-phase component $\operatorname{Re}[K()]$ and an out-of-phase, $\operatorname{Im}[K()]$ - which emanate from the pile periphery and radiate to infinity with continuously decreasing amplitude. These waves control radiation damping. The first resonance influences exclusively the first mode, while the higher modes contribute only to a monotonic attenuation of soil response with radial distance. An additional increase in frequency $\left(\omega_{2}<\omega<\omega_{3}\right)$ yields $q_{2}=i \bar{q}_{2}$, which indicates that the second mode contributes additionally to the loss of energy through radiation. Likewise, with increasing frequency higher modes contribute gradually to wave propagation $\left(q_{m}\right.$ gradually becomes purely imaginary). The total effect is a superposition of all these waves.

Damped medium (Fig. 5): All curves follow the trend described in Fig. 4 (b). Note that the largest deviation among the damped and the undamped curve for each mode is observed close to the resonant frequency $\omega_{m}$, and is becoming more significant at higher modes. For $\omega<\omega_{m}$ the effect of material damping is minor and the curves practically coincide.

Based on Eq. (13) and considering the variation in time the soil response can be expressed as $u_{r, \theta m} \propto \sum U_{r, \theta}(r, \omega) \Phi(z) e^{i \omega t}$. For an undamped soil layer $\left(\beta_{s}=0\right)$ the following cases may be examined:
a) for $\omega<\omega_{m}$, the term $U_{r, \theta} m$ is real valued
b) at $\omega=\omega_{m}$ (resonances), $U_{r, \theta m}=1$
c) for $\omega>\omega_{m}$, the term $U_{r, \theta} m$ is complex valued

Case (a): When $\omega<\omega_{m}$, the energy transmitted from pile oscillations to the soil is stored in terms of dynamic deformations.

Case (b): At resonant frequency $\omega=\omega_{m}$, no spatial variation in motion in $r$ direction for the $m$-th mode exists. Since $U_{r, \theta m}\left(\omega=\omega_{m}\right)=1$, the system undergoes only harmonic motions (i.e., standing waves in the form of $\left.\Phi_{m}(z) e^{i \omega t}\right)$ and the contribution of the $m$-th mode to the soil vibration can be expressed using the simplified expression provided in Eq. (29).

Case $(c)$ : When $\omega>\omega_{m}$, travelling waves develop in the soil and loss of energy occurs (radiation damping). 
Note that in presence of soil material damping $\left(\beta_{s} \neq 0\right)$ and for frequencies $\omega<\omega_{m}$ loss of energy is due to soil material damping.

\section{Plane strain model}

The basic assumption of the plane strain model is that all derivatives with respect to the vertical coordinate are zero, thus no vertical and shear strains develop on the plane perpendicular to the pile axis and only an incompressible horizontal soil slice of the soil medium is considered in the analysis [1]. This model can be viewed as mathematically accurate for an infinitely-long pile embedded in a half space and subjected to uniform lateral displacement along its whole length [32].

The Baranov-Novak soil restraining action can be expressed through a complex valued reaction factor $R^{*}[32]$ :

$$
R^{*}=s^{2} \frac{4 K_{1}(q) K_{1}(s)+s K_{1}(q) K_{0}(s)+q K_{0}(q) K_{1}(s)}{q K_{0}(q) K_{1}(s)+s K_{1}(q) K_{0}(s)+q s K_{0}(q) K_{0}(s)}
$$

where $s$ and $q$ are dimensionless frequency parameters

$$
s=\frac{i \mathrm{a}_{0}}{2 \sqrt{1+2 i \beta_{s}}} \text { and } q=\frac{s}{\eta_{s}}
$$

and $\mathrm{a}_{0}=\omega d / V_{s}$ is a dimensionless frequency and $\eta_{s}$ is obtained from Eq. (18).

Parameter $R^{*}$ is constant with depth and independent of the conditions at the boundaries of the soil layer. Therefore, it cannot capture the layer resonances and exhibits an asymptotic behavior for $\omega \rightarrow 0$ (Fig. 8). In the low-frequency range the soil reaction factor decreases rapidly with decreasing frequency and becomes zero at $\omega=0$. Accordingly, the model cannot capture static stiffness. This deficiency has been identified in earlier studies [4], [16].

Evidently, this is not the case for a pile of finite length embedded in a soil stratum overlying a stiff base. In this study, the soil reaction varies with depth and its variation depends on the boundary conditions at the two ends of the pile. Furthermore, it is sensitive to the natural frequencies of the soil layer. The profile of soil reaction in terms of dynamic stiffness and damping will be examined in a companion paper. Note that the present solution can be reduced to the plane strain model [4] by eliminating the variation of soil displacement components in the vertical direction (i.e., setting $a=0$ in Eqs $6 \mathrm{c}$ and 7). 
Despite its simplicity, the plane strain model yields realistic predictions for frequencies beyond cutoff (see ensuing discussion). Thus, it can be viewed as a special case of the proposed more complete solution, restricted to frequencies beyond cutoff. This is an inherent weakness of the plane strain model, its assumptions being valid only after wave propagation initiates in the medium $\left(\mathrm{a}_{0}>\mathrm{a}_{\text {cutoff }}\right)$.

Note that soil reaction derived from the Baranov-Novak plane strain model is expressed as $p(\omega)=\pi G_{s}^{*} R^{*}$. This is essentially the complex Winkler modulus (dynamic Winkler spring stiffness and dashpot) for laterally loaded piles, and will be investigated in a follow up paper.

\section{Numerical Results}

\section{$\underline{7.1 \text { Static conditions }}$}

The effects of pile length, or equivalently the soil layer thickness, and the selected soil mode on the static soil reaction factor are investigated in Fig. 6. It is shown that for a given $L / d$, higher values of $R_{m}$ correspond to higher modes. This trend is more pronounced for short piles $(L / d<10)$ where the reaction factor pertaining to the 10-th mode is approximately five times higher than the value for the 1 -st mode. Comparison with the early solution of Nogami and Novak [4] shows that the results of that study are always higher than those obtained from the proposed model. The deviation is stronger with decreasing pile length and higher modes - the maximum deviation being observed for $L / d=5$ and $m=10$.

In Fig. 7 the effect of Poisson's ratio on the static reaction factor is investigated for two selected modes. It is shown that higher values of $v_{s}$ always correspond to higher $R_{m}$ for the range of pile lengths considered. As anticipated, the lower the Poisson's ratio the better the agreement with the predictions of the earlier study ( [4]).

\subsection{Dynamic regime}

Results for the soil reaction factor in the dynamic regime obtained from the proposed solution are presented in Figs 8 - 14. The solution of Nogami and Novak [4] is added for comparison. Different representations of the soil reaction factor and frequency are employed, which shed light into the physics of the problem. Some general trends are observed: For each mode $m$, the dynamic soil reaction factor (i.e., real part of soil reaction) decreases with increasing frequency up to the $m$-th resonance, while for the same frequency range, damping (i.e., imaginary part of soil reaction) is practically unaffected by frequency and depends solely on the soil material damping (since only "weak" travelling waves associated with the $m$-th mode develop in 
the medium). At $m$-th resonance the dynamic reaction $R_{m}$ attains a local minimum, which is associated with a distinct jump in damping due to energy radiation, as horizontally travelling waves emerge in the soil medium.

The variation of the real and the imaginary part of dynamic soil reaction with frequency for the first five modes pertaining to a long pile is shown in Fig. 8. The frequency is normalized by the first resonant frequency of the system, $\omega_{1}$. A strong dependence of stiffness (i.e., real part of soil reaction) on the oscillation mode is observed below the resonant frequency, while at the same frequency range the damping (i.e., imaginary part of soil reaction) is practically unaffected by frequency and is controlled by soil material damping. With increasing frequency the dynamic reaction factor becomes gradually independent of soil mode, with all curves practically converging to a single curve at high frequencies. As anticipated, results from the study of Nogami and Novak [4] are always higher than those of the proposed model.

An alternative representation of the results presented in Fig. 8 is shown in Fig. 9, where the dynamic soil reaction factor is normalized by its static value $(\omega=0)$, and the loss of energy (imaginary part) is normalized by twice the real part. It is observed that this type of representation cannot capture the difference between Nogami and Novak [4] and the proposed solution. This indicates that the dynamic modifier expressed by the ratio $R_{m}(\omega) / R_{m}(\omega=0)$ is essentially identical in the two solutions and thus independent of the compressibility of the soil medium in the vertical direction controlled by coefficient $\eta_{s}$.

A perhaps better representation for frequencies in the range $0<\omega<\omega_{m}$ is illustrated in Fig. 10, where the frequency is normalized by a different value for each mode, namely, the $m$-th resonant frequency for the $m$-th propagating mode. It is shown that for dynamic soil reaction all results collapse to a single curve. Also, damping is practically constant and controlled by soil material damping.

The combined effect of soil material damping, pile length (layer thickness) and mode of vibration is investigated in Fig. 11. For a given $L / d$ higher values of soil material damping correspond to higher values of the dynamic soil reaction factor $R$. This trend is reversed for damping since $\beta$ is not a dashpot coefficient but a dimensionless performance index expressed as the ratio of the imaginary part of the complex stiffness $\left[\operatorname{Imaginary}\left(R^{*}\right)\right]$ divided by twice its real part $(2 R)$ (Eq. 27). Considering that the imaginary part of the stiffness is practically unaffected by soil material damping for a given pile or soil thickness (i.e., constant $L / d$ or $H / d$ ), the behavior of $\beta$ is governed by the dynamic stiffness $R$ in the denominator. Thus, higher dynamic stiffness curves correspond to lower damping ratios. In addition, the effect of damping is stronger for short piles $(L / d=5)$ in the high frequency range and for higher modes (e.g., $m=3$ ). At resonance ( $\omega=\omega_{1}$ for the 1 -st mode, $\omega=5 \omega_{1}$ for the 3 -rd mode) the dynamic reaction $R_{m}$ attains a minimum value, 
with the stronger drop pertaining to the lowest material damping. Note that for the extreme case of zero material damping the drop is maximum and $R_{m}$ would reach zero.

The effect of mode number on dynamic soil reaction and damping for a short $(L / d=10)$ and a long $(L / d=50)$ pile in an undamped soil medium is presented in Fig. 12 (a) and (d). Note that different normalization parameters are used for the frequency below and beyond resonance: $\mathrm{a}_{0}=\omega d / V_{s}$ being the familiar dimensionless frequency parameter and $\mathrm{a}_{\text {cutoff }, m}=\omega_{m} d / V_{s}$ being the cutoff frequency of each mode or $m$-th resonance. For frequencies below $m$-th resonance all curves in Fig. 12 (a) start from unity, as the dynamic stiffness is normalized with its static value $(\omega=0)$, and decreases monotonically with frequency. It is shown that higher modes are associated with a higher decrease in stiffness. This effect is more pronounced for short piles. Over the same frequency range, damping is independent of frequency (Fig. 12b) and practically equals the soil material damping, i.e., all damping curves converge to a single curve before resonance. Beyond the cut-off frequency, waves start to propagate in the medium resulting in a sudden increase in damping (Fig. 12b). It is shown that the dynamic stiffness becomes insensitive to the soil thickness $H$ (Fig. 12a). This is an anticipated behavior, since the waves emitted from the periphery of the oscillating pile tend to spread out in a horizontal manner without regard for the vertical dimension ( [19], [16]). This wave radiation pattern explains the very good agreement observed between the plane strain model and the more rigorous solution.

Figures 12 (c) and (d) depict the dimensionless soil reaction impedances and suggest that: (i) below cut-off $\left(\omega<\omega_{m}\right)$ spring and dashpot are best represented in the forms $R_{m}(\omega) / R_{m}(\omega=0)$ and $\beta$, as functions of $\mathrm{a}_{0} / \mathrm{a}_{\text {cutoff,m }}$; (ii) beyond cut-off $\left(\omega>\omega_{m}\right)$, stiffness is best represented in the form $R_{m}(\omega)$ and both parameters as a function of the dimensionless frequency function $\left(a_{0}^{2}-a_{c u t o f f, m}^{2}\right)^{1 / 2}$. Note that the latter parameter has been successfully employed in the representation of the vertical soil reaction in high frequencies for the case of an axially-loaded pile in a homogeneous stratum [27], but has not been explored in the lateral mode.

The effect of pile slenderness - or soil layer thickness - on soil reaction factor of a damped medium is presented in Fig. 13. The numerical results are based on the dimensionless frequencies introduced in Fig. 12. Only the first mode is taken into account. For frequencies below cutoff, longer piles or thicker strata always correspond to higher values of dynamic soil reaction factor; whereas for the damping coefficient all curves practically converge. For frequencies beyond cutoff all curves converge into a single curve and the soil reaction can be well captured by the plane strain model.

The effect of Poisson's ratio on dynamic soil reaction and damping ratio in the dynamic regime is investigated in terms of the 1 st mode for a short and a long pile and shown in Fig. 14. For frequencies below 
cutoff, variations in Poisson's ratio do not affect stiffness and damping (Figs. 14 a1, b1, c1). For frequencies beyond cutoff, a minor effect of Poisson's ratio on damping is observed at high frequencies (Fig. 14 c2). On the other hand, the results in Fig. 14 (a2) indicate that the effect is strong on dynamic soil reaction, with higher values of $v_{s}$ resulting in higher stiffness. However, an alternative representation of soil reaction shown in Fig. 14 (b2) (dynamic soil reaction is normalized by static value) shows that the influence of Poisson's ratio on "pure" dynamic stiffness is observed only in the high frequency range and can be considered negligible. The strong variations depicted in Fig. 14 (a2) are attributed to the effect of Poisson's ratio on the static soil reaction.

\section{Simplified expression for $\boldsymbol{R}^{*}(\omega)$}

Results obtained from the proposed model as shown in Fig. 14 (a2) are plotted against predictions from the plane strain model in Fig. 15 (a). Evidently, the plane strain model yields unrealistic results for the incompressible case, the parabolic-like decreasing trend can be attributed to a "trapped mass" effect which is not exhibited in the 3D model [33]. This is an inherent weakness of the simpler model and it is suggested to use results obtained from Eq. (31) only for Poisson's ratios less than 0.4.

In this study, a simplified expression for the soil reaction factor is presented. Taking the limit $v_{s} \rightarrow 0.5 \mathrm{Eq}$. (31) attains the asymptotic form

$$
R^{*}(\omega)=s\left(s+4 \frac{K_{1}(s)}{K_{0}(s)}\right)
$$

which does not yield realistic results (Fig. 15a). Herein, the above expression is modified to account for Poisson's ratio. This can be achieved by introducing a new semi empirical expression for the parameter $s$

$$
s=\frac{i \mathrm{a}_{0}}{2 \sqrt{1+2 i \beta_{s}}\left(\eta_{s}\right)^{\chi}}
$$

which encompasses the compressibility parameter $\eta_{s}$ and the empirical parameter $\chi$ which is a function of the Poisson's ratio only (Table 1). Results from Eqs (34) and (35) are compared with those obtained from the proposed model in Fig. 15 (b). The very good agreement in the range $0.1<v_{s}<0.4$ and the improved performance over the plane strain model for $v_{s}=0.5$ can hardly be overstated.

\section{Infinitely - long pile}


The above solution can be readily extended to model an infinitely-long pile embedded in a half space. Although idealized, this limit case is useful since the solution for infinitely long piles is independent of the thickness of the soil layer $(H / d)$ and covers all flexible piles (actual pile length > active length). In this light, one may assume that the solution for piles of finite length is useful only for low values of pile slenderness $L / d$. A discussion on active pile length is provided in Randolph [34], Velez et al. [35] and Syngros [20].

For an infinitely long pile no distinct eigenmodes exist. Instead, the solution for soil displacements and stresses is obtained by integrating over all possible values of $\alpha$. The soil reaction may be written as follows:

$$
p(z, \omega)=\pi G_{s}^{*} \int_{0}^{\infty} R_{\alpha}^{*}(\omega) W(\omega) \Phi(z) d a
$$

where $R_{\alpha}^{*}$ is the complex valued soil reaction factor (Eq. 25)

$$
R_{a}^{*}=s^{2} \frac{\eta_{\sigma}^{2}\left[2 K_{1}\left(s \eta_{s}\right)+s \eta_{s} K_{0}\left(s \eta_{s}\right)\right] K_{1}(s)+\eta_{s}^{2}\left[2 K_{1}(s)+s K_{0}(s)\right] K_{1}\left(s \eta_{s}\right)}{s K_{0}(s) K_{1}\left(s \eta_{s}\right)+s \eta_{s} K_{0}\left(s \eta_{s}\right)\left[s K_{0}(s)+K_{1}(s)\right]}
$$

where $s=q d / 2$, and $q$ obtained from Eq. (7). As for the case of a soil layer $R_{a}(\omega)=\operatorname{Real}\left(R_{a}^{*}\right)$ is the dynamic storage stiffness and $\beta_{a}=\operatorname{Imaginary}\left(R_{a}^{*}\right) / 2 \operatorname{Real}\left(R_{a}^{*}\right)$ defines an equivalent damping ratio.

A three-dimensional representation of the dynamic soil reaction and damping ratio as a function of the excitation frequency and the parameter $\alpha$ is shown in Figs 16 and 17. Note that each curve corresponds to a soil layer of finite length that is characterized by a fictitious natural frequency, yet these natural frequencies are suppressed upon integration in terms of Eq. (35). In both graphs all natural frequencies are placed on a straight line for which $a d=\omega d / V_{s}$.

\section{Conclusions}

An approximate three - dimensional solution is developed for the dynamic reaction of a homogeneous half space and a soil layer over a rigid base to the laterally oscillating pile. Contrary to the classical elastodynamic equations which cannot be solved analytically, the proposed approach allows a closed form solution to be obtained both for an infinitely long pile and a pile of finite length.

The main findings of this study can be summarized as follows:

a) The main assumption adopted in this study is that the vertical dynamic normal stress $\sigma_{z}$ is zero. This approximation is compatible with the presence of a free surface and leads to a small, yet finite 
vertical soil displacement. This overcomes the sensitivity of earlier models to Poisson's ratio for nearly incompressible media.

b) From the interference of upward and downward traveling disturbances associated with each mode $m$ in the soil layer a standing wave emerges. The total response is a superposition of $m$-th standing waves, which is a spatially varying (stands still and oscillates left and right), but not propagating disturbance (not a travelling), and naturally no loss of energy in terms of radiation damping occurs. The drop in dynamic soil reaction and sudden increase in damping are associated with the natural frequencies of the soil layer and the emergence of travelling waves in the horizontal direction. The wave propagation phenomenon was thoroughly discussed in this study and was investigated by means of a frequency spectrum using the first five modes.

c) A new dimensionless incremental frequency parameter $\left(a_{0}^{2}-a_{\text {cutoff }, m}^{2}\right)^{1 / 2}$ was introduced for describing the soil reaction in the high frequency range $\left(a_{m}>a_{c u t o f f, m}\right)$. It was shown that this representation allows the reaction factor to exhibit the same behavior regardless of actual soil layer thickness (or pile length), value of Poisson's ratio, soil material damping and oscillation mode.

d) It was found that the dynamic soil reaction factor below cutoff frequency is best normalized by the corresponding static stiffness $(\omega=0)$ as a function of dimensionless frequency ratio $\mathrm{a}_{0} / \mathrm{a}_{\text {cutoff,m }}$. Beyond the cutoff frequency, the dynamic stiffness can be best normalized by the soil's shear modulus (no special scheme is needed) and is best expressed as a function of incremental frequency $\left(a_{0}^{2}-a_{c u t o f f, m}^{2}\right)^{1 / 2}$ (Fig. 12). The only exception exists when investigating the effect of $v_{s}$ on dynamic soil reaction, where a normalization with the static value is required over the whole range of frequencies (Fig. 14). These properties stem from the dependence of the solution on the cutoff frequency of each mode and the gradual transformation of the wave field emitted from the pile with increasing frequency beyond resonance, from three-dimensional to two-dimensional (plane strain).

e) It was also observed that with increasing frequency the plane strain solution converges to the more rigorous solution. However, significant discrepancies in stiffness appear, especially for short piles, for frequencies below cutoff and for the particular case of $v_{s}=0.5$ in the high frequency range.

f) A simple, improved expression for determining dynamic soil reaction based on an asymptotic form of the classic plane strain solution which takes into account the compressibility of the soil was presented in Eqs (33) and (34).

g) A solution for the dynamic soil reaction factor and the corresponding damping ratio were derived for the case of an infinitely long pile in a half space. This is achieved by expressing the soil response 
in terms of integrals instead of Fourier series, as in the case of piles of finite length (Eq. 35). Soil reaction was then derived as a superposition of modular soil reactions for all possible depths of soil layers to form a half space. 


\section{Notation}

$A, B, C, D \quad$ integration constants depending on boundary conditions and type of loading

$d \quad$ pile diameter

$E_{S} \quad$ Young's modulus of soil

$G_{S}, G_{S}^{*} \quad$ real- and complex-valued shear modulus of soil

$H \quad$ thickness of soil layer

$\widetilde{m}_{s} \quad$ mass of an infinitesimal soil element in cylindrical coordinates $\left(r d r d \theta d z \rho_{s}\right)$

$p \quad$ horizontal soil reaction to pile motion

$q_{m} \quad$ soil frequency-dependent parameter

$r \quad$ radial or horizontal coordinate

$t \quad$ time variable

$V_{S}, V_{S}^{*} \quad$ real-valued and complex-valued soil shear wave propagation velocity

$w \quad$ lateral pile displacement

$W_{m} \quad$ pile response Fourier coefficients

$u_{r}, u_{\theta} \quad$ soil displacement components (radial, tangential)

$z \quad$ vertical coordinate

\section{Greek Symbols}

\begin{aligned} \multicolumn{1}{l}{$a, a_{m}$} & positive real-valued number (eigenvalues) \\ $\beta_{s} &$ soil material damping \\ $\beta_{r} &$ radiation damping \\ $\eta_{s}, \eta_{\sigma} &$ compressibility parameters \\ $v_{s} &$ soil Poisson's ratio \\ $\rho_{s} &$ soil mass density \\ $\sigma_{r}, \sigma_{\theta}, \sigma_{z} &$ normal stresses in soil \\ $\tau_{\theta z}, \tau_{z r}, \tau_{r \theta} &$ shear stresses in soil \\ $\mathbf{\Phi}, \Psi &$ potential functions \\ $\omega &$ cyclic excitation frequency \\ $\omega_{m}, \omega_{n} &$ m-th resonant and natural frequency \\ $\nabla &$ Laplacian operator \end{aligned}




\section{Appendix A}

In cylindrical coordinates, normal strains are written in terms of displacements via the well-known equations

$\varepsilon_{r}=\frac{\partial u_{r}}{\partial r}$

$\varepsilon_{z}=\frac{\partial u_{z}}{\partial z}$

$\varepsilon_{\theta}=\frac{u_{r}}{r}+\frac{1}{r} \frac{\partial u_{\theta}}{\partial \theta}$

Based on the assumption of zero normal stress $\sigma_{z}$, normal strain $\varepsilon_{z}$ is written as

$\varepsilon_{z}=-K_{0}\left(\varepsilon_{r}+\varepsilon_{\theta}\right)$

and normal stresses $\sigma_{r}$ and $\sigma_{\theta}$ can be expressed in terms of normal strains $\varepsilon_{r}$ and $\varepsilon_{\theta}$ in the form

$\sigma_{r}=G_{s}^{*}\left[\eta_{\sigma}^{2}\left(\varepsilon_{r}+\varepsilon_{\theta}\right)-2 \varepsilon_{\theta}\right]$

$\sigma_{\theta}=G_{s}^{*}\left[\eta_{\sigma}^{2}\left(\varepsilon_{r}+\varepsilon_{\theta}\right)-2 \varepsilon_{r}\right]$

where $K_{0}=v_{s} /\left(1-v_{s}\right)$ is the coefficient of lateral pressure at rest for an elastic material depending solely on Poisson's ratio; $\eta_{\sigma}$ is also a function of Poisson's ratio, given by Eq. (26) in the present formulation and Eq. (18) in the work by Tajimi.

Shear strains are written in terms of displacements as

$\gamma_{\theta z}=\frac{\partial u_{\theta}}{\partial z}+\frac{1}{r} \frac{\partial u_{z}}{\partial \theta}$

$\gamma_{r z}=\frac{\partial u_{r}}{\partial z}+\frac{\partial u_{z}}{\partial r}$

$\gamma_{r \theta}=\frac{1}{r} \frac{\partial u_{r}}{\partial \theta}+\frac{\partial u_{\theta}}{\partial r}-\frac{u_{\theta}}{r}$

Shear stresses $\tau$ in terms of shear strains $\gamma$

$$
\begin{aligned}
& \tau_{\theta z}=G_{s}^{*} \gamma_{\theta z} \\
& \tau_{r \theta}=G_{s}^{*} \gamma_{r \theta}
\end{aligned}
$$


$\tau_{z r}=G_{s}^{*} \gamma_{z r}$

In light of Eq. (A.2a), the derivatives $\partial \tau_{r z} / \partial z$ and $\partial \tau_{\theta z} / \partial z$ attain the alternative forms

$$
\begin{aligned}
& \frac{\partial \tau_{r z}}{\partial z}=G_{s}^{*}\left(\frac{\partial^{2} u_{r}}{\partial z^{2}}+\frac{\partial \varepsilon_{z}}{\partial r}\right) \\
& \frac{\partial \tau_{\theta z}}{\partial z}=G_{s}^{*}\left(\frac{\partial^{2} u_{\theta}}{\partial z^{2}}+\frac{1}{r} \frac{\partial \varepsilon_{z}}{\partial \theta}\right)
\end{aligned}
$$

which depend solely on the horizontal displacement components $u_{r}$ and $u_{\theta}$.

Substituting Eqs (A.2), (A.3), (A.6) into the equilibrium equations (1), yields the Navier equations (2). 


\section{References}

[1] V. Baranov, "On the calculation of an embedded foundation. Voprosy Dinamiki i Prochnosti.," polytech.Inst.Riga, Latvia., vol. 14, pp. 195-209, 1967.

[2] M. Novak, "Dynamic Stiffness and Damping of Piles," Canadian Geotechnical Journal, vol. 11, no. 4, pp. 574-598, 1974.

[3] H. Tajimi, "Dynamic analysis of a structure embedded in an elastic stratum," in 4th World Conference on Earthquake Engineering, Santiago, Chile, 1969.

[4] T. Nogami and M. Novak, "Resistance of soil to a horizontally vibrating pile," Earthquake Engineering and Structural Dynamics, vol. 5, no. 3, pp. 249-261, 1977.

[5] M. Saitoh and H. Watanabe, "Effects of flexibility on rocking impedance of deeply embedded foundations," J. Geotech. Geoenvironmental Eng., vol. 130, no. 4, pp. 438-445, 2004.

[6] A. Veletsos and A. Younan, "Dynamic soil pressures on rigid cylindrical vaults," Earthquake Engineering and Structural Dynamics, vol. 23, no. 6, pp. 645-669, 1994.

[7] A. Veletsos and A. Younan, "Dynamic modeling of response of rigid embedded cylinders," J. Eng. Mech., vol. 121, no. 9, pp. 1026-1035, 1995.

[8] K. Graff, Wave Motion in Elastic Solids., Dover Publications, 1975.

[9] A. Eringen and E. S. Suhubi, Elastodynamics: Volume II: Linear Theory, Academic Press, 1974.

[10] H. Matsuo and S. Ohara, "Lateral earth pressures and stability of quay walls during earthquakes," in 2nd World Conference on Earthquake Engineering, Tokyo, Vol I, 1960.

[11] G. Anoyatis, "Contribution to kinematic and inertial analysis of piles by analytical and experimental methods," PhD thesis, University of Patras, Rio, Greece, 2013. 
[12] J. Roesset, "Stiffness and damping coefficients of foundations," in ASCE Geotechnical Engineering Division National Convention, 1980.

[13] R. Dobry, E. Vicente, M. O'Rourke and M. Roesset, "Horizontal stiffness and damping of single piles," Journal of Geotechnical and Geoenvironmental Engineering, vol. 108, no. 3, pp. 439-759, 1982.

[14] G. Gazetas and R. Dobry, "Horizontal response of piles in layered soil," J. Geotech. Engrg. Div., vol. 110, no. 1, pp. 20-40, 1984a.

[15] G. Gazetas and R. Dobry, "Simple Radiation Damping Model for Piles and Footings," J. Eng. Mech., vol. 110, no. 6, pp. 937-956, 1984b.

[16] G. Mylonakis, "Elastodynamic model for large-diameter end-bearing shafts," Journal of the Japanese Geotechnical Society: Soils and Foundations, vol. 41, no. 3, pp. 31-44, 2001 b.

[17] H. Tajimi, "Seismic Effects on Piles," in Spec. Session 10, IX, ICSMFE, Tokyo, 1977.

[18] M. Pender, "Aseismic pile foundation design analysis," Bull. New Zealand Nat. Soc. for Earthquake Engrg., vol. 26, no. 1, pp. 49-160, 1993.

[19] G. Gazetas and G. Mylonakis, "Seismic soil-structure interaction: New evidence and emerging issues.," Spec. Publ. No. 75, ASCE, pp. 1110-1174, 1998.

[20] C. Syngros, "Seismic response of piles and pile supported bridge piers evaluated through case histories," Ph.D. Thesis, City University of New York, 2004.

[21] W. D. Guo, Theory and practice of pile foundations, London: CRC Press, 2012.

[22] M. Shadlou and S. Bhattacharya, "Dynamic stiffness of pile in a layered elastic continuum," Geotechnique, vol. 64, no. 4, pp. 303-319, 2014.

[23] T. Akiyoshi, "Soil-pile interaction in vertical vibration induced through a frictional interface," Earthquake Engineering and Structural Dynamics, vol. 10, no. 1, pp. 135-148, 1982. 
[24] K. Chau and X. Yang, "Nonlinear interaction of soil-pile in horizontal vibration," J. Eng. Mech., vol. 131, no. 8, pp. 847-858, 2005.

[25] C. Latini, V. Zania and B. Johannesson, "Dynamic Stiffness and damping of foundations for jacket structures," in 6th International Conference on Earthquake Geotechnical Engineering, Christchurch, New Zealand, 2015.

[26] X. Karatzia, P. Papastylianou and G. Mylonakis, "Horizontal Soil Reaction of a Cylindrical Pile Segment with a Soft Zone," J. Eng. Mech., vol. 140, no. 10, pp. 1061 -, 2014.

[27] G. Anoyatis and G. Mylonakis, "Dynamic Winkler modulus for axially loaded piles," Geotechnique, vol. 62, no. 6, pp. 521-536, 2012.

[28] C. R. Wylie and L. C. Barrett, Advanced engineering mathematics, McGraw-Hill, 1995.

[29] R. Butterfield and P. K. Banerjee, "The elastic analysis of compressible piles and pile groups," Geotechnique, vol. 21, no. 1, pp. 43-60, 1971.

[30] A. P. S. Selvadurai and R. K. N. D. Rajapakse, "On the load transfer from a rigid cylindrical inclusion into an elastic half space," International Journal of Solids and Structures, vol. 21, no. 12, pp. 12131229, 1985.

[31] R. W. Clough and J. Penzien, Dynamics of Structures, New York: McGraw Hill, 1993.

[32] M. Novak, T. Nogami and F. Aboul-Ella, "Dynamic soil reactions for plane strain case," Journal of the Engineering Mechanics Division, vol. 104, no. 4, pp. 953-959, 1978.

[33] F. E. Richart, J. R. Hall and R. D. Woods, Vibrations of soils and foundations, Prentice Hall, 1970.

[34] M. Randolph, "The response of flexible piles to lateral loading," Geotechnique, vol. 31, no. 2, pp. 247-259, 1981.

[35] A. Velez, G. Gazetas and R. Krishnan, "Lateral dynamic response of constraint-head piles," Journal of Geot. Eng., vol. 109, no. 8, pp. 1063-1081, 1983. 
[36] G. Mylonakis, "Winkler modulus for axially loaded piles," Geotechnique, vol. 51, no. 5, pp. 455-461, 2001a.

[37] M. Novak and T. Nogami, "Soil-pile interaction in horizontal vibration," Earthquake Engineering and Structural Dynamics, vol. 5, no. 3, pp. 263-281, 1977. 


\section{Tables}

Table 1: Values of empirical parameter $\chi$ as function of Poisson's ratio

\begin{tabular}{|c|c|c|c|c|c|}
\cline { 2 - 6 } \multicolumn{1}{c|}{} & \multicolumn{5}{|c|}{ Poisson's ratio $v_{s}$} \\
\hline$\chi$ & 0.1 & 0.2 & 0.3 & 0.4 & 0.5 \\
\hline$\chi$ & 5 & 4 & 3 & 2 & 1 \\
\hline
\end{tabular}




\section{FIGURES}

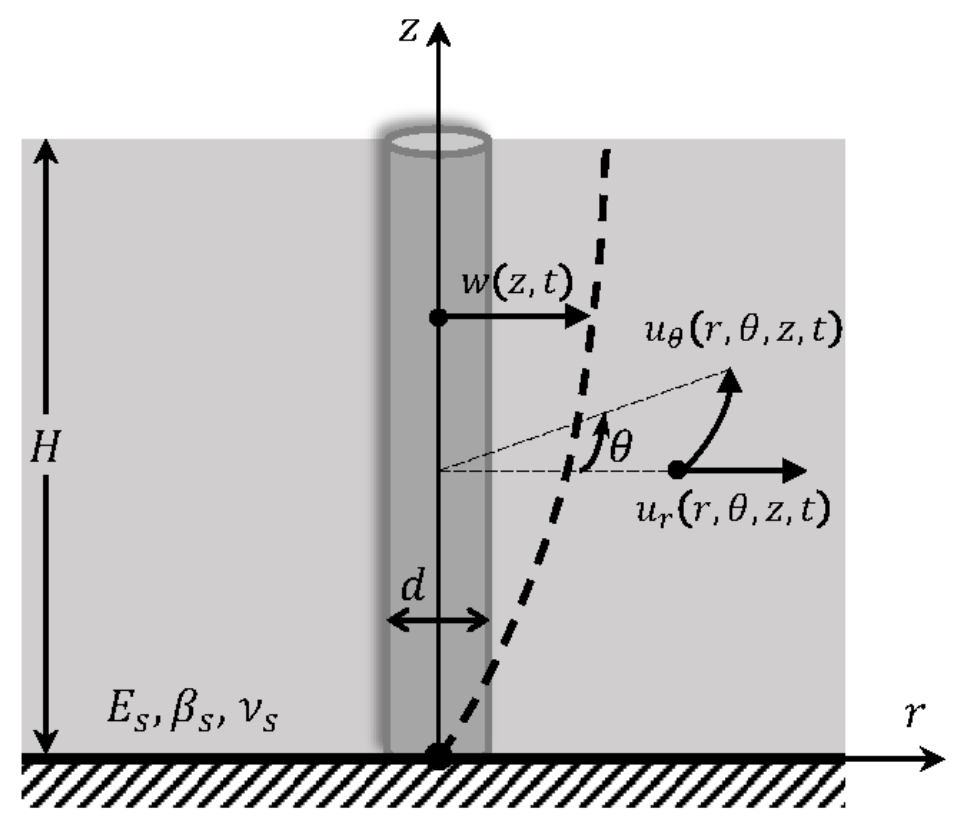

Fig. 1: Problem considered: Harmonically excited cylindrical vertical pile embedded in soil stratum overlying a rigid base
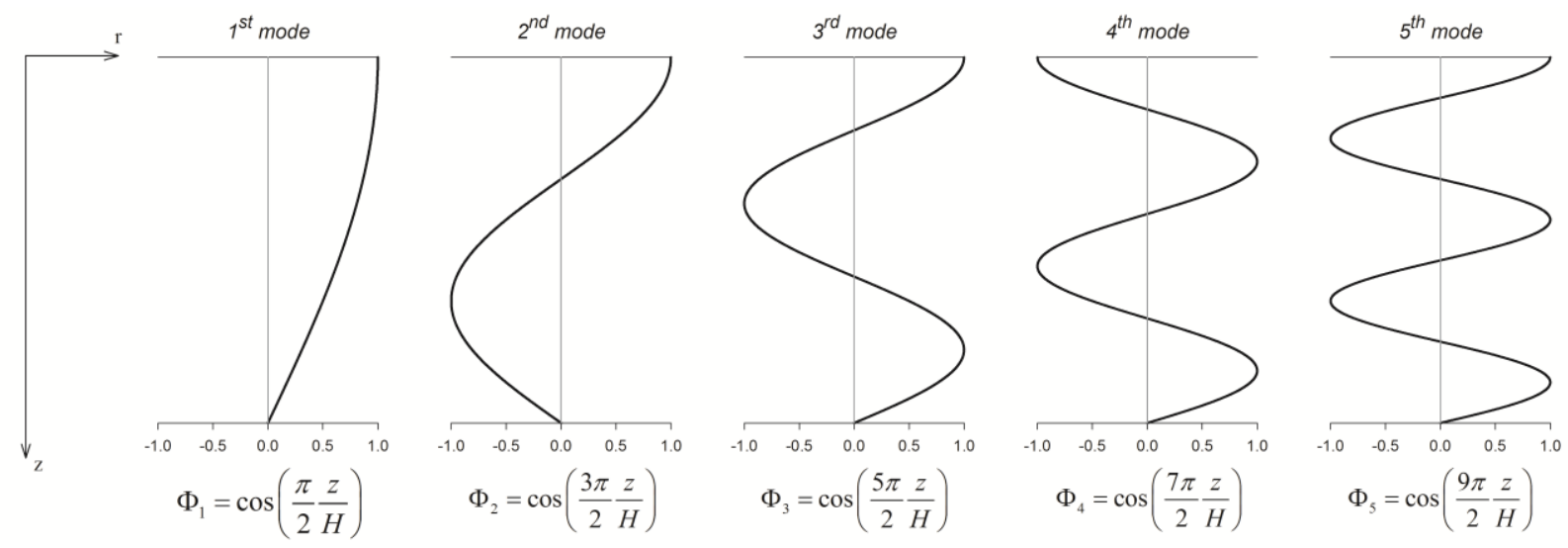

Fig. 2: First five modes of soil layer due to lateral pile motion 


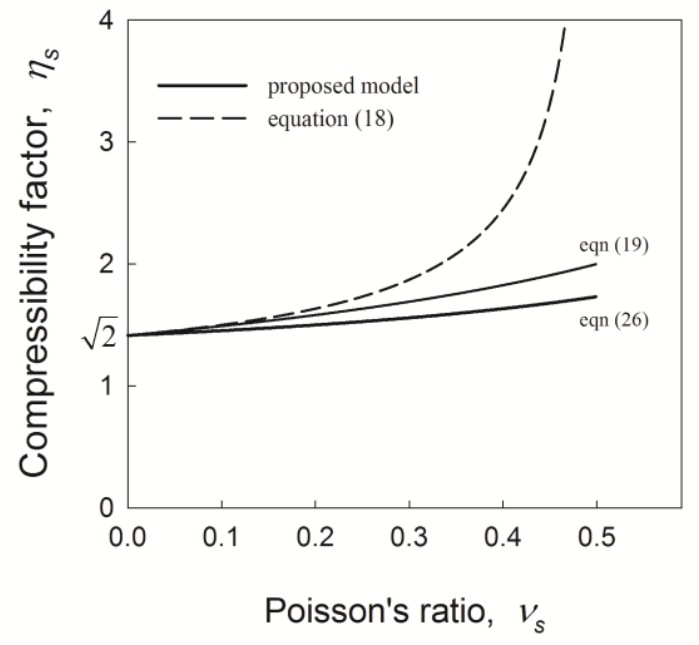

Fig. 3: Variation of compressibility factor with Poisson's ratio

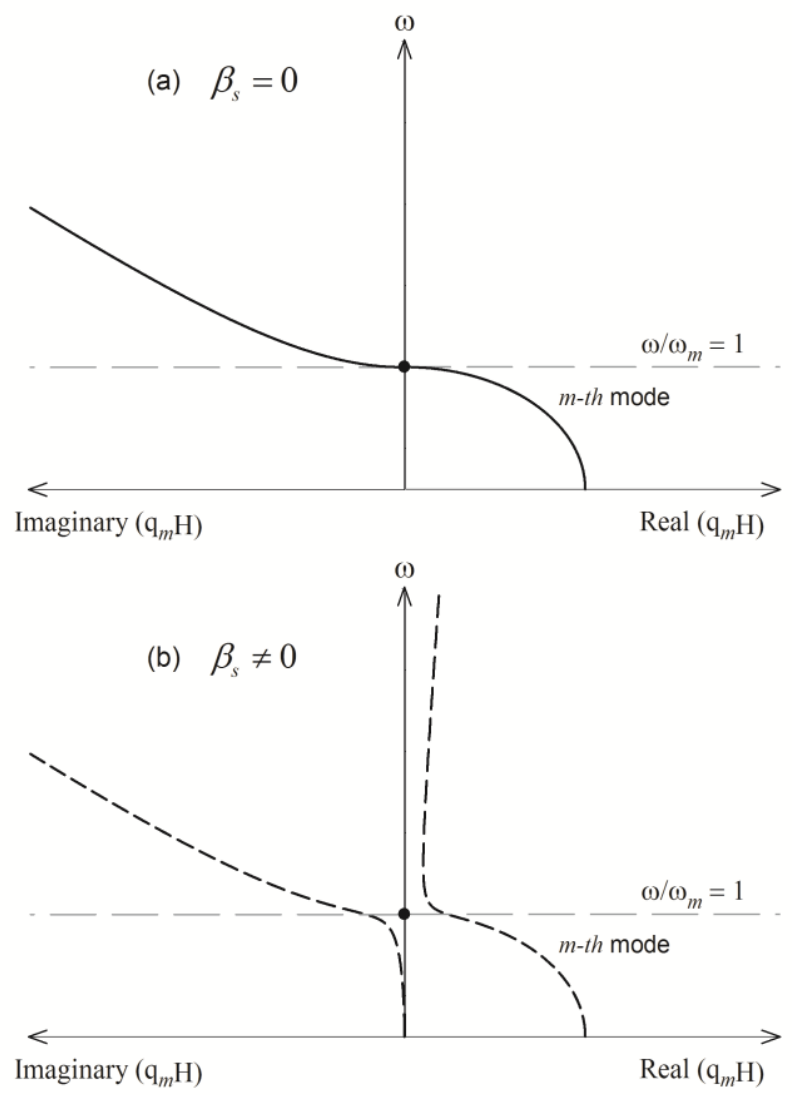

Fig. 4: Schematic representation of the frequency spectrum in the m-th mode 


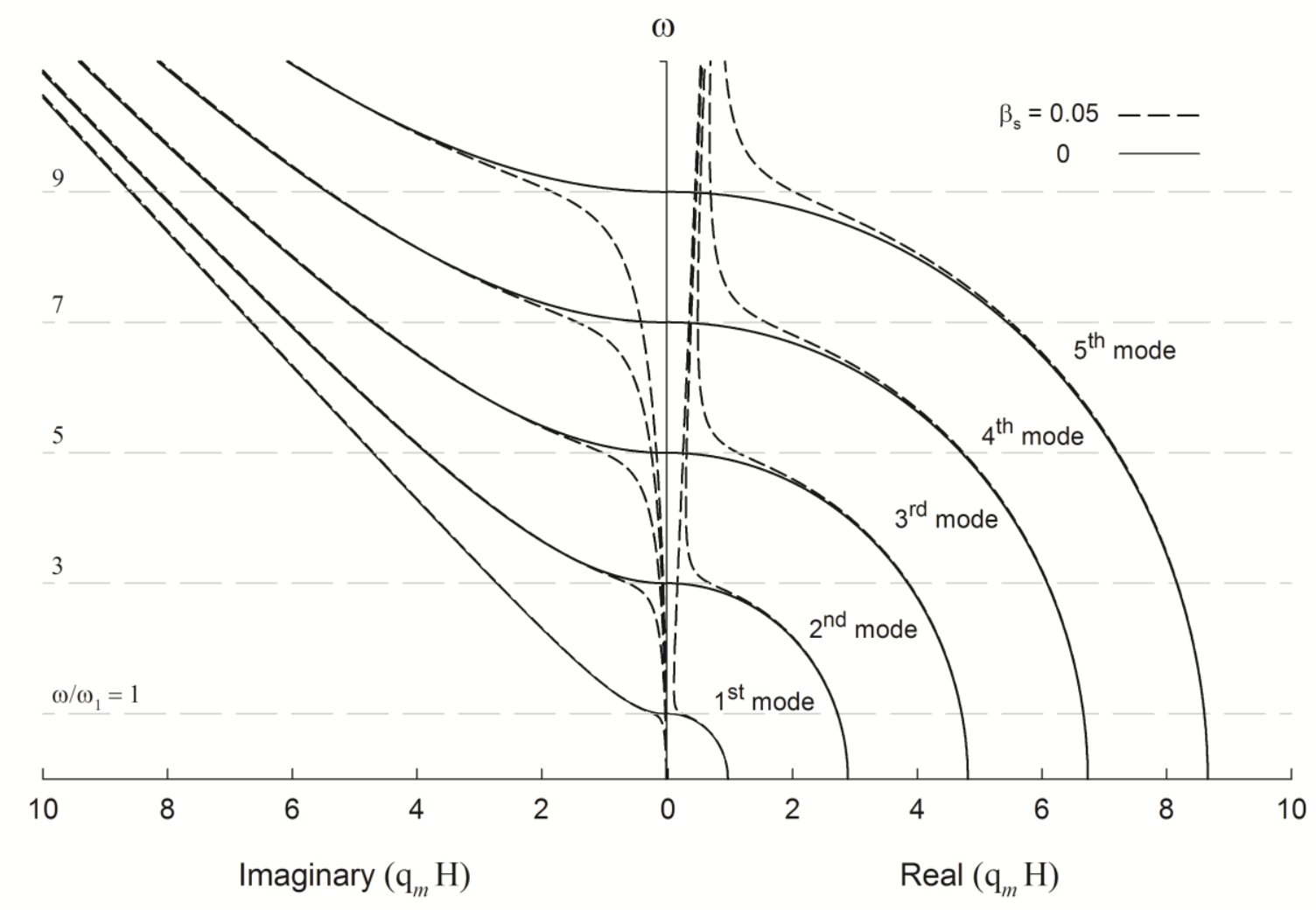

Fig. 5: Frequency spectrum for the five first modes in an undamped and a damped soil medium 


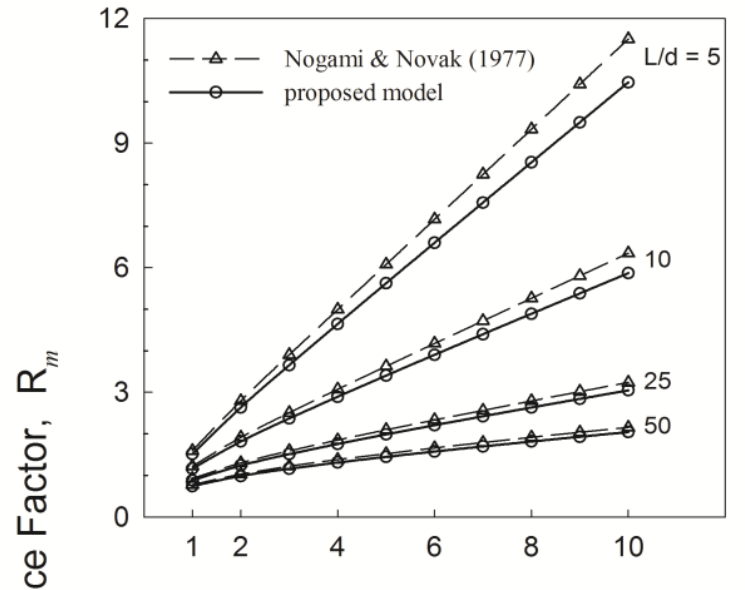

(a) Mode, $m$

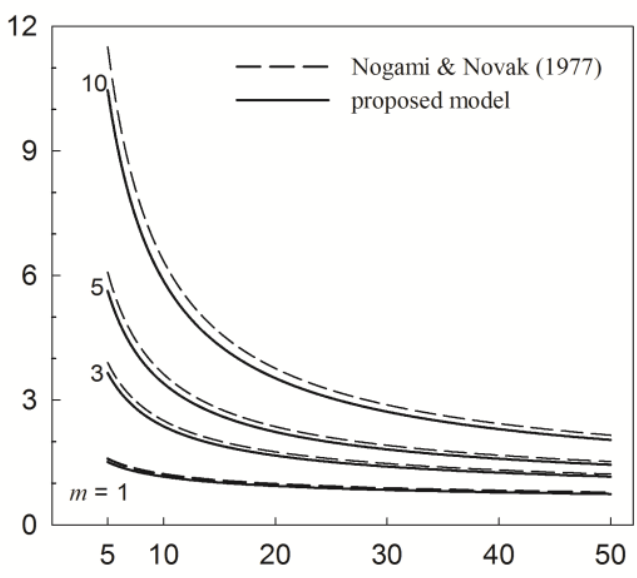

(b) Pile slenderness, L/d

Fig. 6: Effect of modes and pile slenderness on static soil resistance factor 


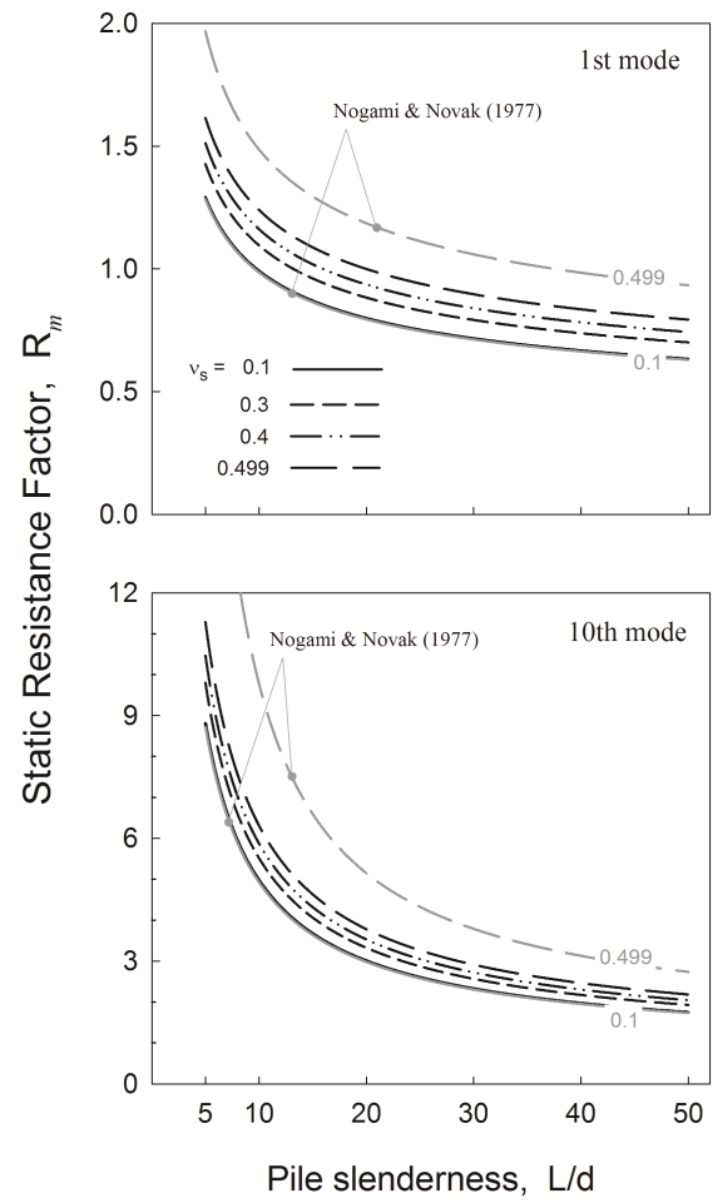

Fig. 7: Effect of Poisson's ration on static soil resistance factor 

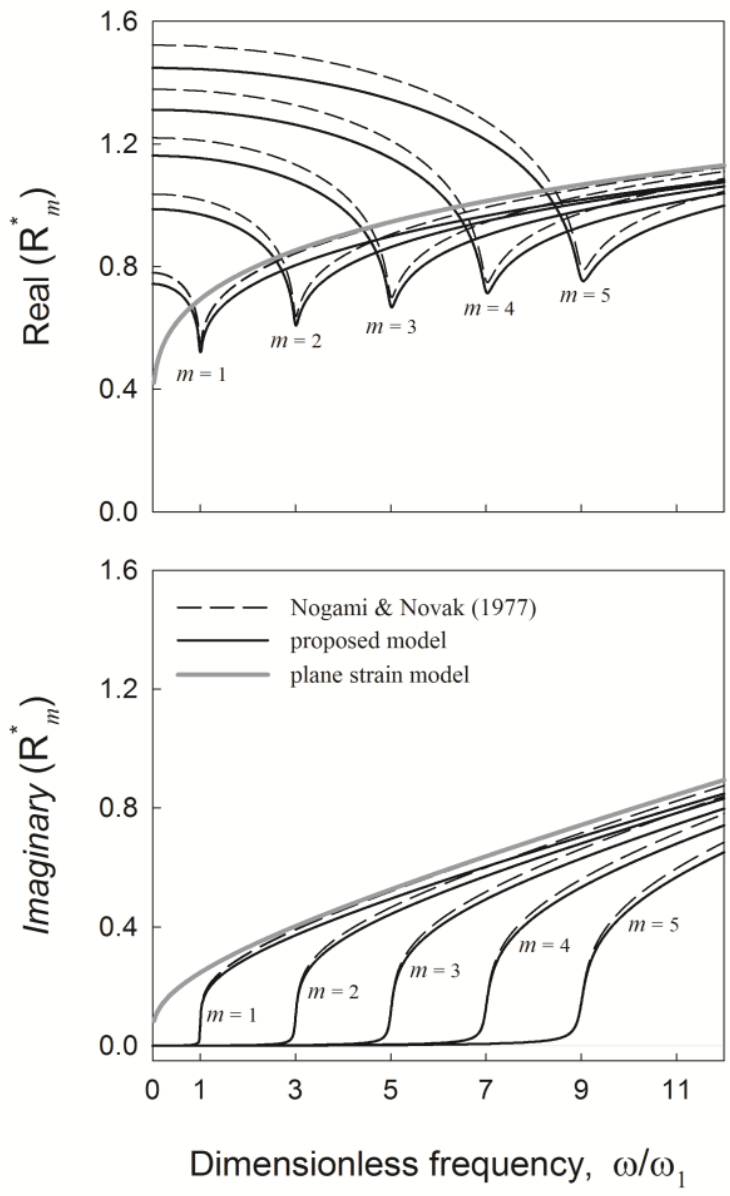

Fig. 8: Variation of real and imaginary part of soil reaction with frequency for selected modes; Comparison with Nogami and Novak [4] and plane strain model; $H / d=50, \beta_{s}=0.01$ 

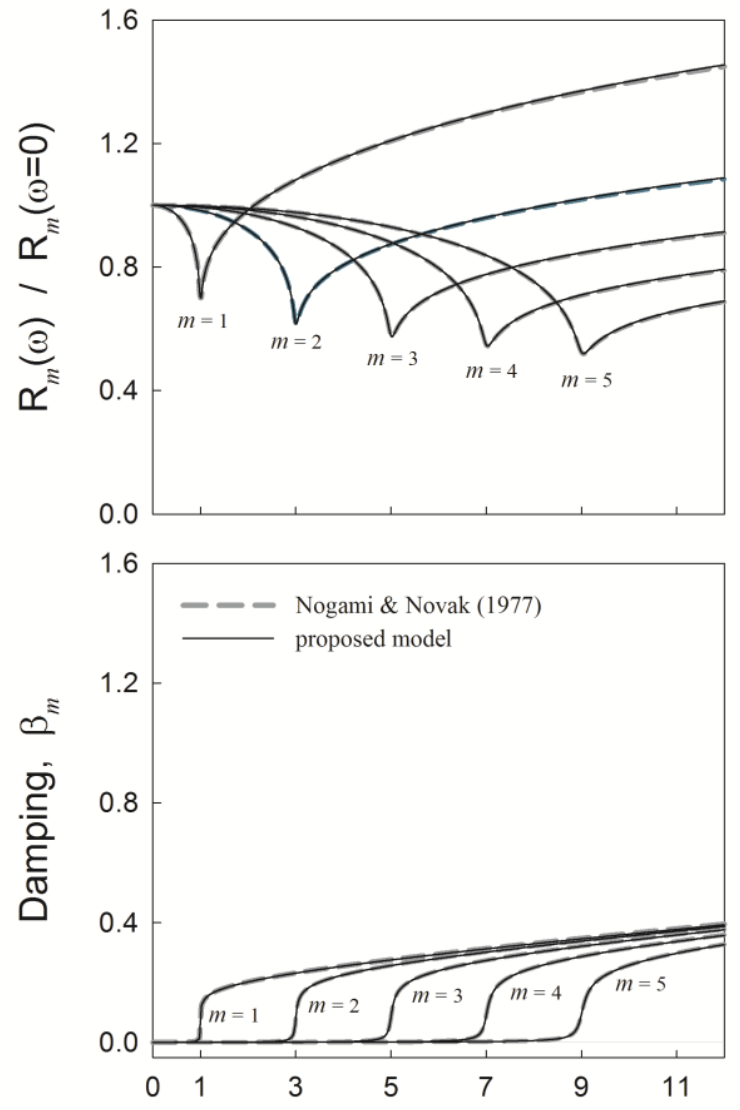

Dimensionless frequency, $\omega / \omega_{1}$

Fig. 9: Variation of dynamic soil reaction $R_{m}(\omega)$ and damping with frequency for selected modes; Comparison with Nogami and Novak [4]; $H / d=50, \beta_{s}=0.01$ 

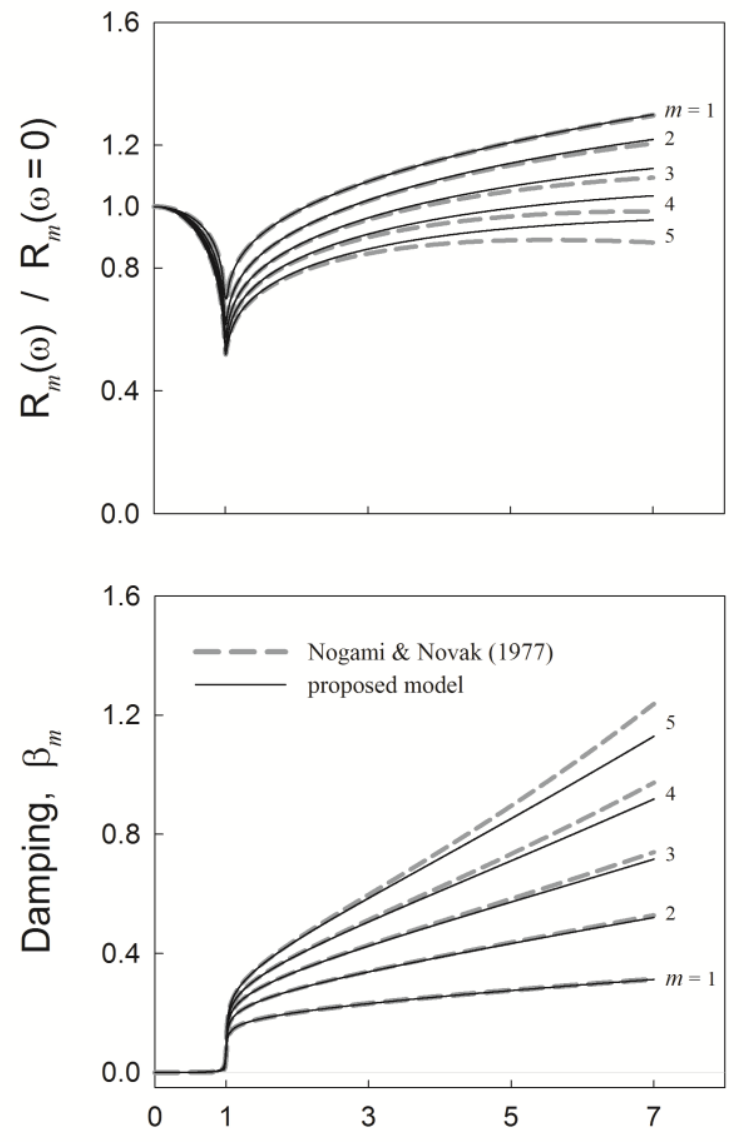

Dimensionless frequency, $\omega / \omega_{m}$

Fig. 10: Variation of dynamic soil reaction $R_{m}(\omega)$ and damping with frequency for selected modes; Comparison with Nogami and Novak [4]; $H / d=50, \beta_{s}=0.01$ 

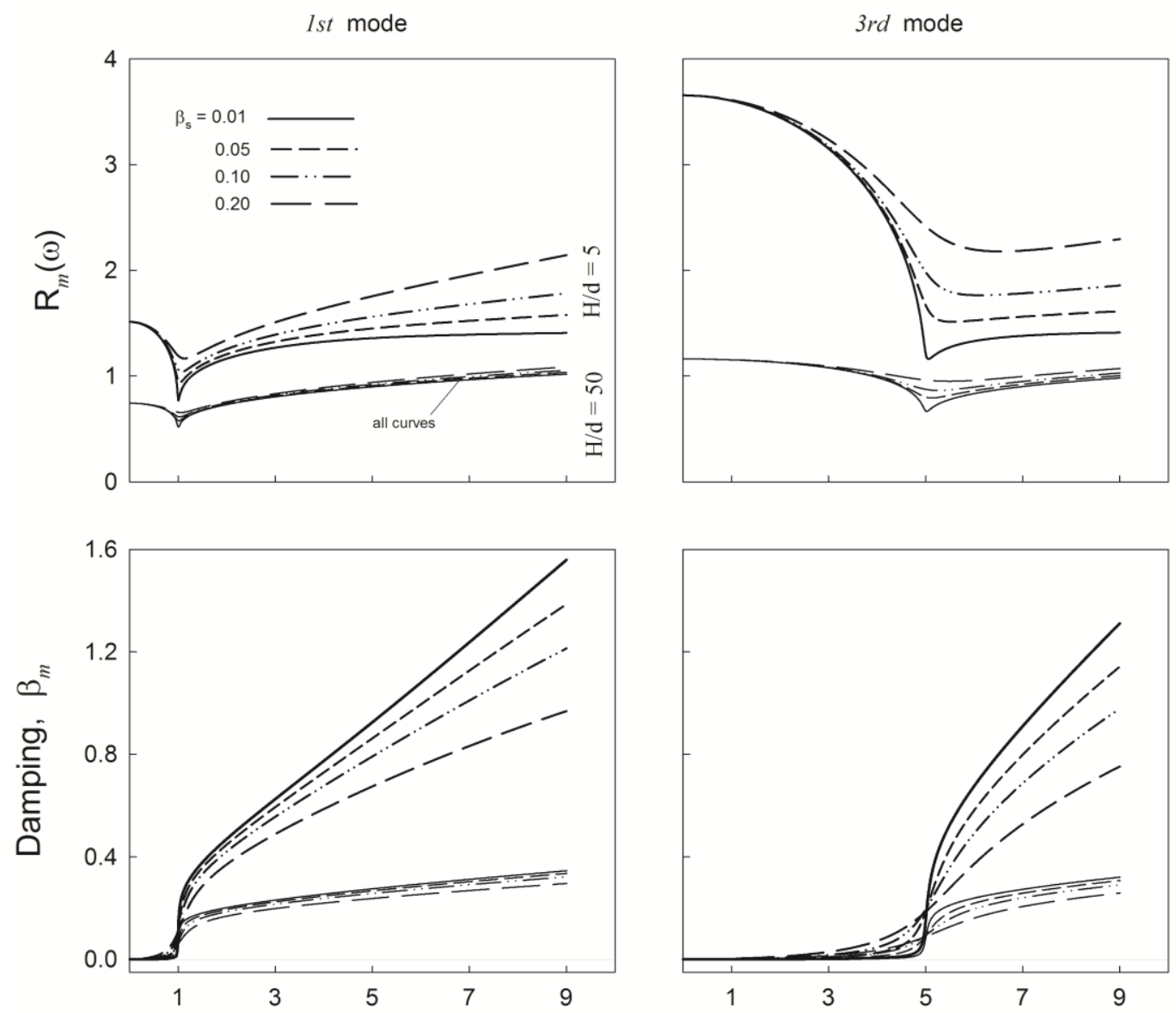

Dimensionless frequency, $\omega / \omega_{1}$

Fig. 11: Variation of dynamic soil reaction $R_{m}(\omega)$ and damping with frequency for selected modes, pile slenderness and material damping 

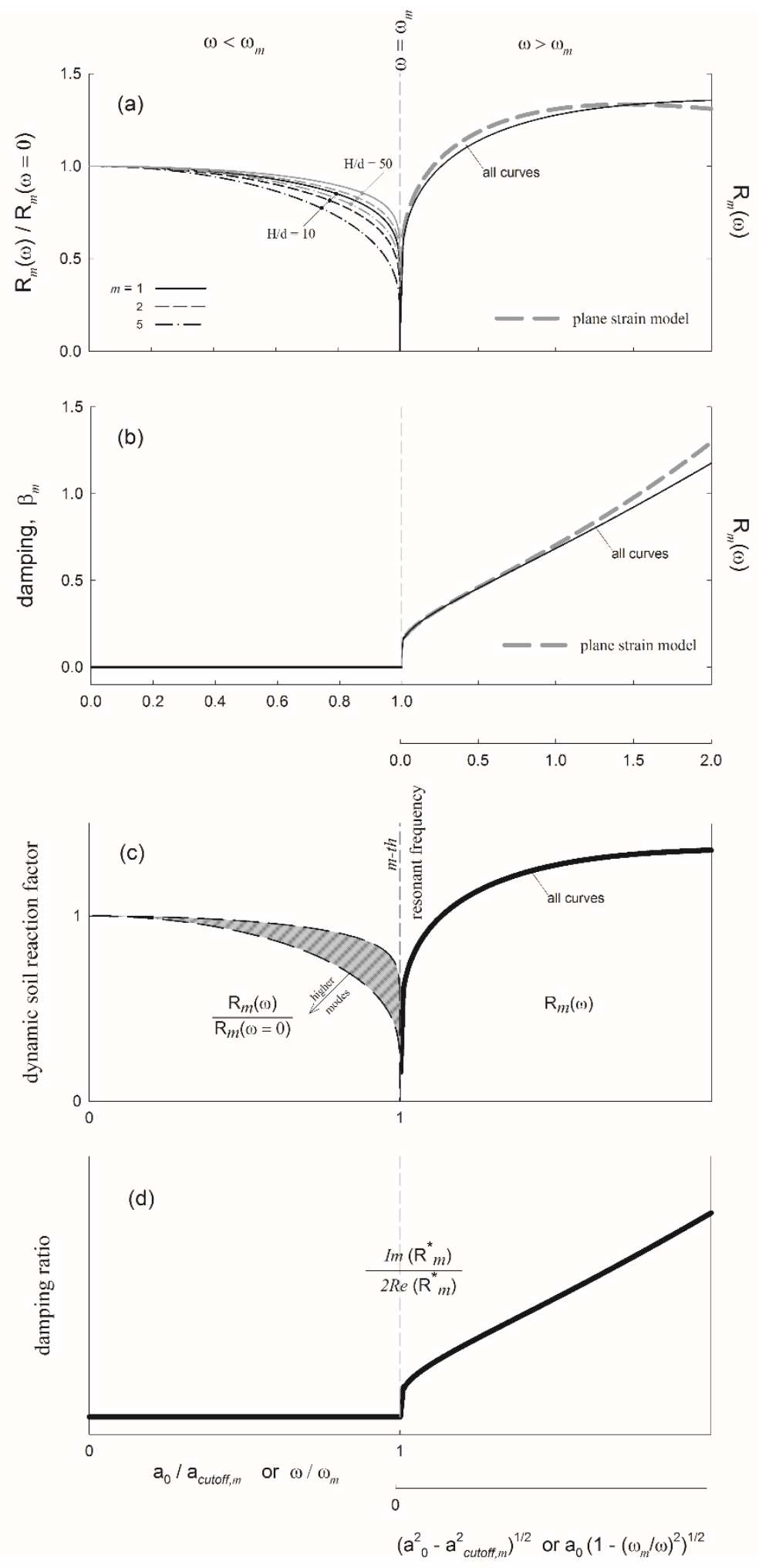

Fig. 12: (a), (d) Effect of number in mode on soil reaction in dynamic regime $\left(\beta_{s}=0\right)$;

(b), (c) Schematic representation of variation of dynamic soil reaction and damping ratio with novel dimensionless frequency parameters 
$\omega<\omega_{1}$
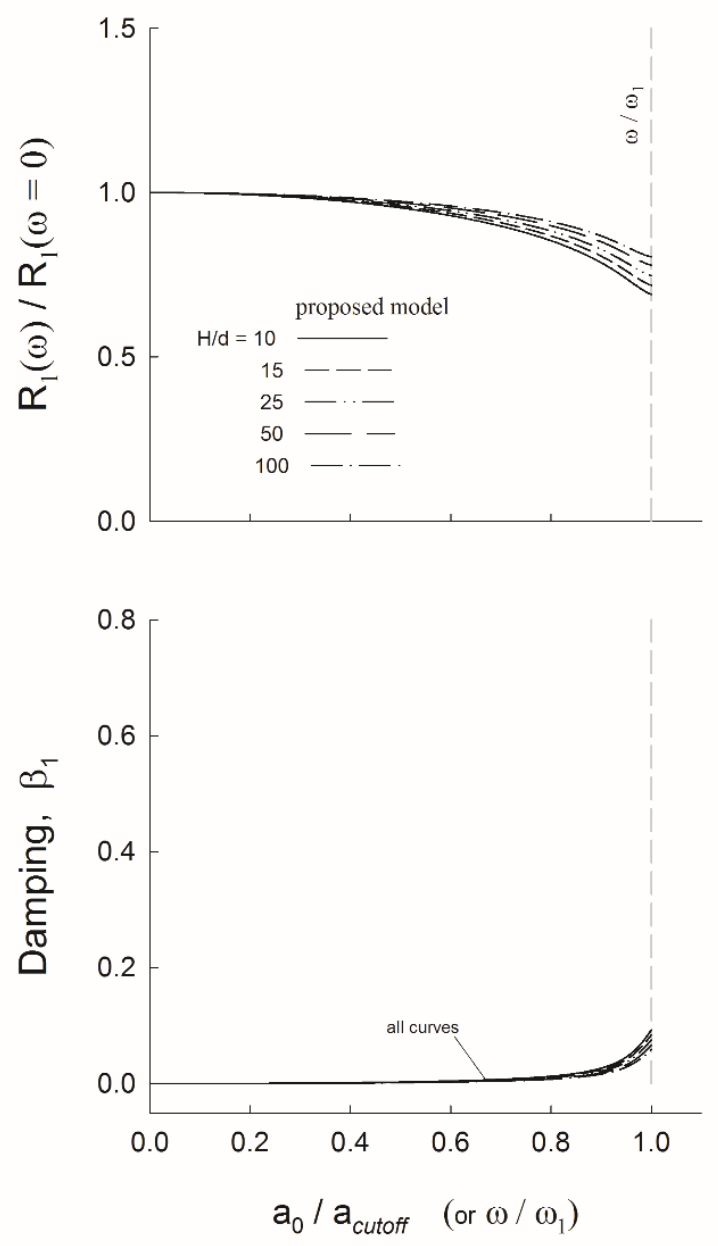

$\omega>\omega_{l}$
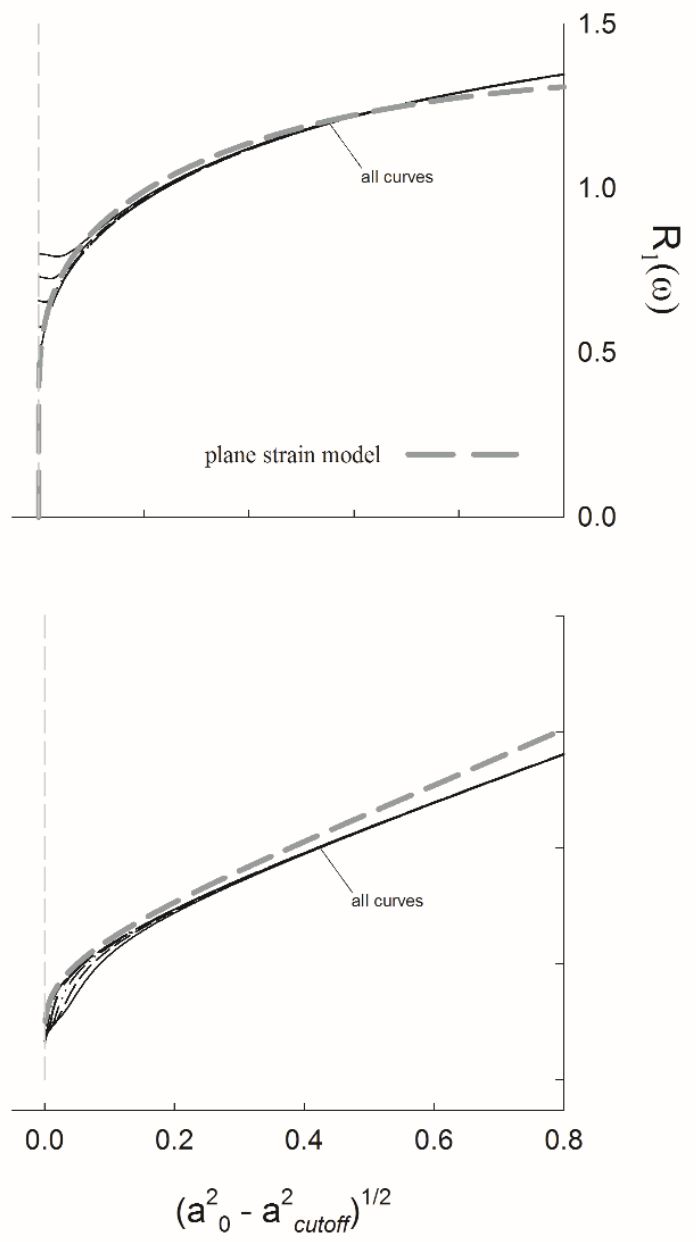

Fig. 13: Effect of pile slenderness on soil reaction in dynamic regime; $\beta_{s}=0.05, v_{s}=0.4$ 

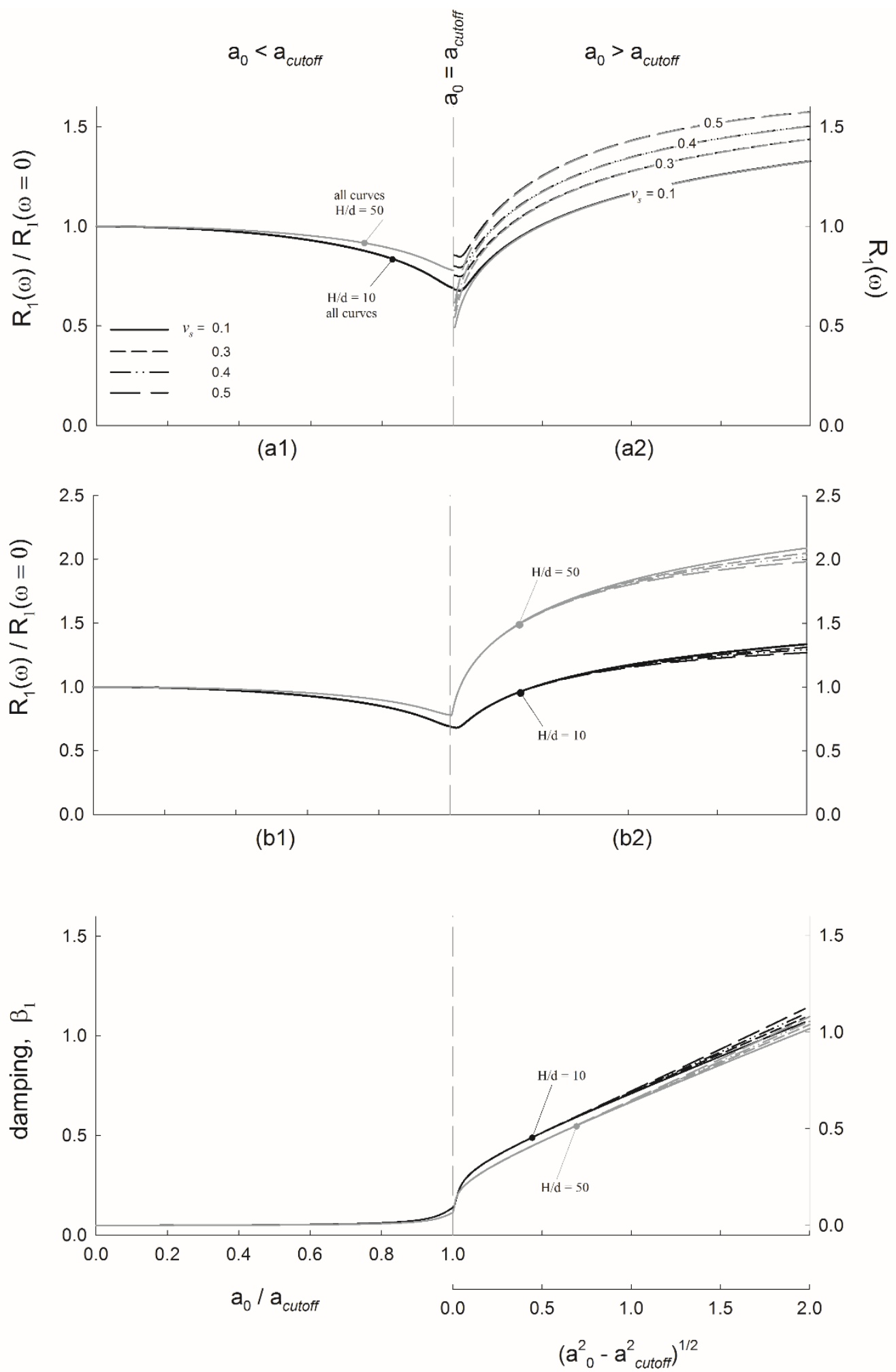

(c1)

(c2)

Fig. 14: Effect of Poisson's ratio on dynamic soil reaction and damping; $\beta_{s}=0.05$ 

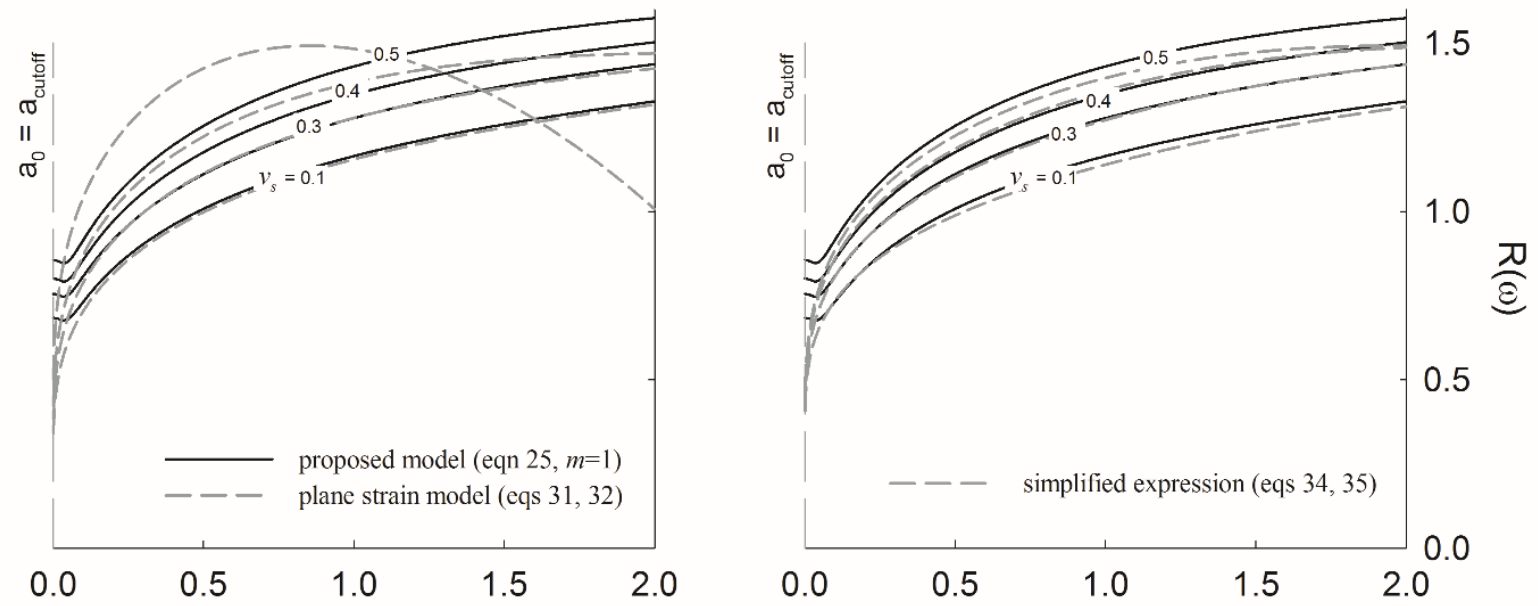

$$
\left(a_{0}^{2}-a_{\text {cutoff }}^{2}\right)^{1 / 2}
$$

(a)

(b)

Fig. 15: Effect of Poisson's ratio on dynamic soil reaction $R(\omega) ;($ a) comparison with plane strain model; (b) comparison with simplified (improved) expression; $\beta_{s}=0.05$ 


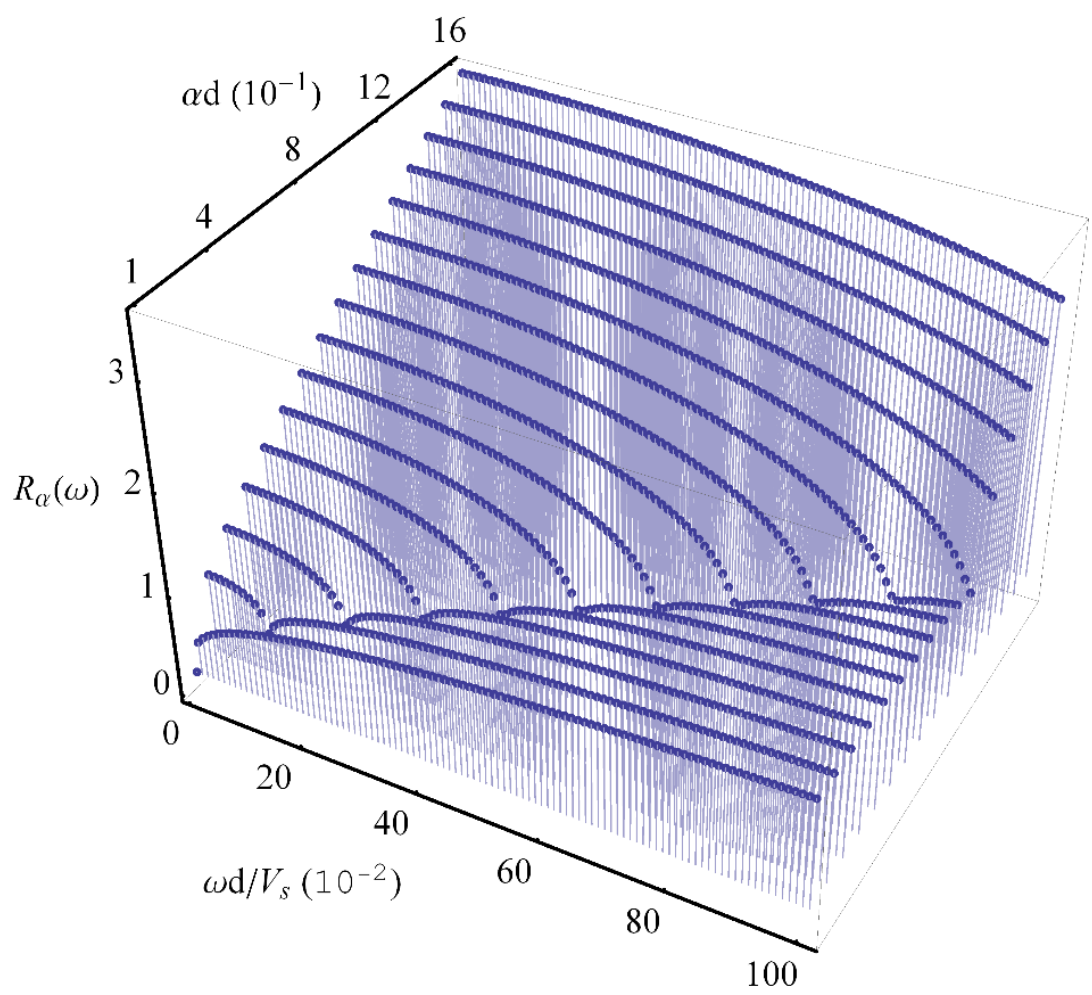

Fig. 16: $3 D$ representation of dynamic soil reaction factor $R_{a}(\omega)$ with frequency and $(\alpha d) ; \beta_{s}=0.01$, $v_{s}=0.4$

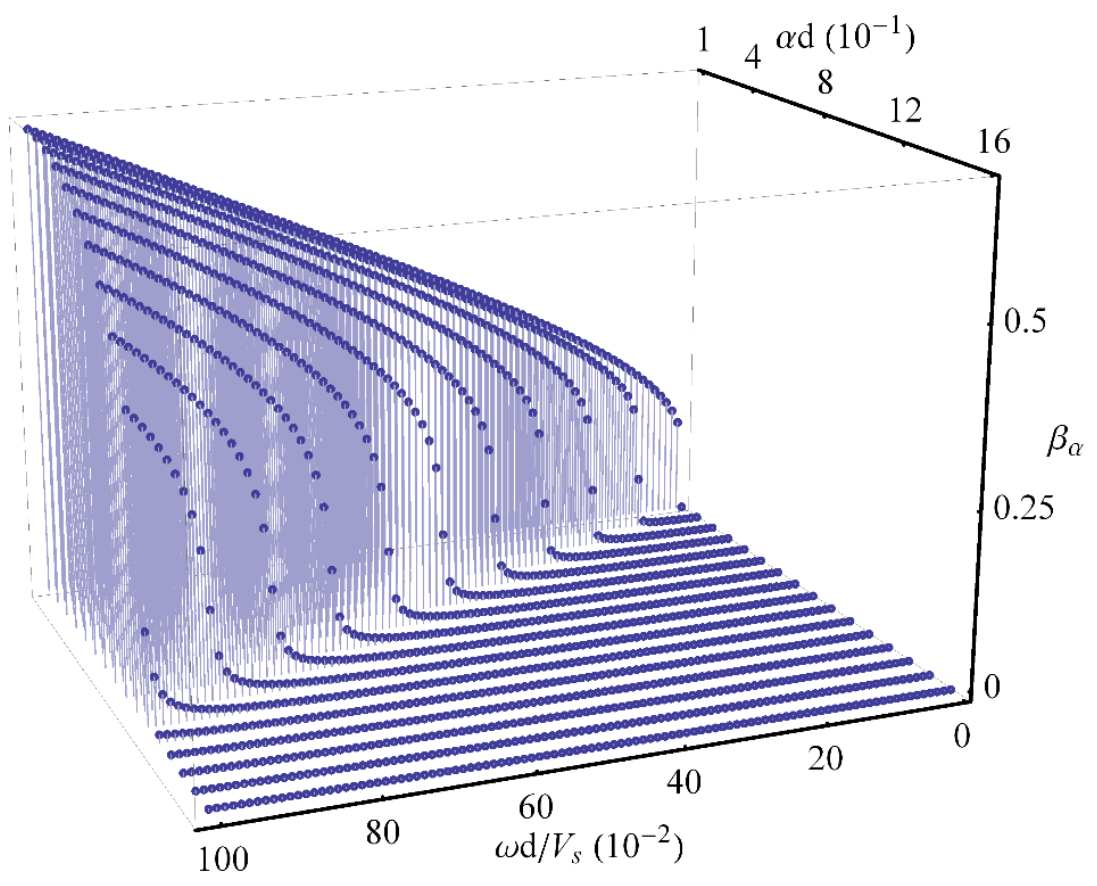

Fig. 17: $3 D$ representation of damping ratio $\beta_{a}$ with frequency and $(\alpha d) ; \beta_{s}=0.01, v_{s}=0.4$ 